\title{
Comparative phylogenomics of the CBL-CIPK calcium-decoding network in the moss Physcomitrella, Arabidopsis, and other green lineages
}

\author{
Thomas J. Kleist ${ }^{1 *}$, Andrew L. Spencley ${ }^{1,2}$ and Sheng Luan ${ }^{1 *}$ \\ ' Department of Plant and Microbial Biology, University of California Berkeley, Berkeley, CA, USA \\ 2 Department of Dermatology, Stanford University, Stanford, CA, USA
}

Edited by:

Rohini Garg, National Institute of Plant Genome Research, India

\section{Reviewed by:}

Caroline Gutjahr, Ludwig Maximilian University of Munich, Germany

Matthew R. Willmann, University of

Pennsylvania, USA

\section{${ }^{*}$ Correspondence:}

Thomas J. Kleist and Sheng Luan, 451 Koshland Hall Berkeley, CA 94720, USA

e-mail: kleist@berkeley.edu; sluan@berkeley.edu
Land plants have evolved a host of anatomical and molecular adaptations for terrestrial growth. Many of these adaptations are believed to be elaborations of features that were present in their algal-like progenitors. In the model plant Arabidopsis, 10 Calcineurin B-Like proteins (CBLs) function as calcium sensors and modulate the activity of 26 CBL-Interacting Protein Kinases (CIPKs). The CBL-CIPK network coordinates environmental responses and helps maintain proper ion balances, especially during abiotic stress. We identified and analyzed CBL and CIPK homologs in green lineages, including CBLs and CIPKs from charophyte green algae, the closest living relatives of land plants. Phylogenomic evidence suggests that the network expanded from a small module, likely a single CBL-CIPK pair, present in the ancestor of modern plants and algae. Extreme conservation of the NAF motif, which mediates CBL-CIPK physical interactions, among all identified CIPKs supports the interpretation of CBL and CIPK homologs in green algae and early diverging land plants as functionally linked network components. We identified the full complement of CBL and CIPK loci in the genome of Physcomitrella, a model moss. These analyses demonstrate the strong effects of a recent moss whole genome duplication: CBL and CIPK loci appear in cognate pairs, some of which appear to be pseudogenes, with high sequence similarity. We cloned all full-length transcripts from these loci and performed yeast two-hybrid analyses to demonstrate CBL-CIPK interactions and identify specific connections within the network. Using phylogenomics, we have identified three ancient types of CBLs that are discernible by $\mathrm{N}$-terminal localization motifs and a "green algal-type" clade of CIPKs with members from Physcomitrella and Arabidopsis.

Keywords: CBL-CIPK, calcium signaling, plant abiotic stress physiology, plant nutrition, evolution, molecular

\section{INTRODUCTION}

Of the events that have shaped our modern biosphere, the colonization of land by the predecessors of modern embryophytes stands out as an evolutionary advent that has profoundly affected our landscape and terrestrial ecology. Land plants arose roughly 450 million years ago from a lineage of multicellular freshwater green algae known as charophytes (Graham, 1996; Lewis and McCourt, 2004). Land plants have elaborated and expanded upon a molecular toolkit present in their charophyte ancestors and thereby developed novel anatomical and molecular adaptations to withstand life on land (Graham, 1996; Kenrick and Crane, 1997; Pittermann, 2010; Timme and Delwiche, 2010). The switch from aquatic to terrestrial growth imposed new and formidable abiotic stresses. Discontinuous access to water combined with labile, often unfavorable ion balances spurred the development of sophisticated mechanisms for the perception of water and ion availability, the communication of this information throughout the plant body, and the coordination of orchestrated responses to these stresses.
Calcium ions play a pivotal role in a host of signal transduction cascades in plants as well as in animals. Tightly localized spikes in cytosolic calcium concentration in response to particular environmental cues have been extensively documented in plant cells and are thought to act as early steps in plant signaling pathways (Gilroy et al., 1993; Evans et al., 2001). These bursts, known as calcium signals, are modulated by channels that allow calcium entry from both outside the cell and inside cellular stores (e.g., the vacuole, endoplasmic reticulum). Calcium signals are decoded by proteins that act as sensors; calcium sensors often contain helix-loop-helix motifs known as EF hands that bind calcium and induce conformational changes to modulate the activity of other proteins or domains (Hrabak et al., 2003; McCormack et al., 2005).

Calcineurin B-Like proteins or CBLs are a family of calcium sensors found in all studied land plants and some chlorophyte green algae (Weinl and Kudla, 2009; Batistic et al., 2011). CBLs are named based on their homology to the B regulatory subunit of the phosphatase calcineurin (Luan et al., 2002). CBLs 
contain four calcium-binding EF hands and typically contain a subcellular localization signal at their N-terminus. The most thoroughly characterized CBLs to date contain a dual lipid modification motif (MGCXXS/T) at their N-terminus that is necessary and sufficient for targeting of fluorescent protein (FP)-fusions to the plasma membrane (Batistic et al., 2008, 2010). Other CBLs are reported to localize to the vacuole, and several of these CBLs contain a distinct N-terminal extension known as the Tonoplast Targeting Sequence (TTS) that targets FP-fusions to the tonoplast (Batistič, 2012; Tang et al., 2012). Uniquely, Arabidopsis CBL10 contains a putative $\mathrm{N}$-terminal transmembrane helix that anchors it to the tonoplast (Kim et al., 2007; Batistic et al., 2010) or plasma membrane (Quan et al., 2007; Ren et al., 2013). Subcellular targeting has been shown to be critical for CBL functionality, and CBLs are responsible for the recruitment and localization of protein partners.

CBLs physically and functionally interact with CBLInteracting Protein Kinases (CIPKs) and modulate their kinase activity (Shi et al., 1999; Batistic et al., 2011). Hence, the CBL-CIPK network serves to decode calcium signals and transmit these signals through reversible protein phosphorylation. CIPKs, also known as SnRK3 proteins, are serine/threonine protein kinases that consist of a $\mathrm{N}$-terminal kinase domain similar to those found in other plant protein kinases and a unique C-terminal regulatory domain that acts as an autoinhibitory domain and mediates interactions with CBLs. CBLs bind to a short, conserved region within the C-terminal autoinhibitory domain of CIPKs known as the NAF or FISL motif (Shi et al., 1999; Albrecht et al., 2001; Guo et al., 2001). In addition to modulating the kinase activity of CIPKs, CBLs are thought to be the sole or primary determinants of CBL-CIPK complex localization, therefore they are thought to act as functional modules (Luan, 2009; Batistic et al., 2011). CBLs are believed to recruit CIPKs, which lack any sort of discernible targeting signals, to these surfaces, possibly in a calcium-dependent manner (Batistič and Kudla, 2009; Batistic et al., 2010).

Initial functional analysis of the CBL-CIPK network came from the genetic identification of the Salt Overly Sensitive (SOS) pathway. Together, CBL4/SOS3 and CIPK24/SOS2 modulate that activity of the plasma membrane $\mathrm{Na}^{+} / \mathrm{H}^{+}$exchanger SOS1. Mutants lacking any component of the Salt Overly Sensitive (SOS) pathway display NaCl-hypersensitive phenotypes (Liu and Zhu, 1998; Liu et al., 2000; Shi et al., 2000). CBL4/SOS3 and CIPK24/SOS2 belong to large proteins families containing 10 CBLs and 26 CIPKs in Arabidopsis and similarly sized families in other angiosperms (Kudla et al., 1999; Kolukisaoglu et al., 2004; Weinl and Kudla, 2009). CBL-CIPK complexes have recently been implicated in sodium, potassium, nitrate, and proton transport (Li et al., 2006; Xu et al., 2006; Ho et al., 2009); therefore the CBLCIPK network is currently thought to be a major regulator of ion homeostasis in angiosperms.

Though CBLs and CIPKs have been discovered among all studied land plants and certain green algal lineages, little is known about the functionality of the CBL-CIPK network outside of angiosperms. As an initial step toward functional analysis of the CBL-CIPK network in an early-diverging land plant, we analyzed the genomic content of CBLs and CIPKs in the model moss Physcomitrella and performed bioinformatic analyses of the CBL and CIPK families with an emphasis on relationships among Physcomitrella and Arabidopsis CBLs and CIPKs. We classified CBLs according to their phylogeny and N-terminal localization motifs and identified three ancient classes of CBLs. Using yeast two-hybrid analyses, we confirmed interactions among CBLs and CIPKs outside of angiosperms and characterized physical interactions among Physcomitrella CBLs and CIPKs. Through phylogenetic analyses, we identified a strongly supported clade that contains all CIPKs identified from green algae and two CIPKs from Arabidopsis and Physcomitrella. Using phylogenomic methods, we seek to characterize patterns of expansion of the CBLCIPK network among land plant lineages to classify CBLs and CIPK in an evolutionarily and functionally meaningful manner to facilitate functional genetic work in early-diverging plants.

\section{MATERIALS AND METHODS HOMOLOG IDENTIFICATION, SEQUENCE ALIGNMENT, AND BIOINFORMATIC ANALYSES}

CBL and CIPK homologs were identified using BLASTp and tBLASTn searches of the Uniprot and the NCBI protein and nucleotide databases, using previously identified CBLs and CIPKs from Arabidopsis as queries. Additional sequences were manually retrieved by annotation from UniProt using the keywords "calcineurin" and "CBL-interacting" (Jain et al., 2009). Genomic loci of CBL and CIPK homologs in Physcomitrella patens were identified in version 1.6 of the Physcomitrella genome, available at http://cosmoss.org (Zimmer et al., 2013). All charophyte CBL and CIPK sequences identified were predicted by assembly of homologous expressed sequence tags (ESTs) from transcriptome-level sequencing of diverse, representative charophyte genera (Timme and Delwiche, 2010; Timme et al., 2012). Other new CBL and CIPK protein sequences were predicted from EST sequences in the NCBI non-redundant (nr) nucleotide database identified by tBLASTn searches. Overlapping ESTs from the same taxa were assembled, and ESTs were translated using Geneious R6 (Biomatters), which was also used for all stages of phylogenetic analyses and figure preparation. Predicted CBL and CIPK homologs were verified by manual inspection of domain architecture and pBLAST searches of the NCBI non-redundant (NR) protein database; all protein sequences included in analyses showed expected domain architecture and yielded top BLASTp hits to previously identified CBLs and CIPKs. CBL and CIPK homologs identified in this study are listed in Supplementary Tables S1, S2, respectively. Protein sequences were aligned using MAFFT (algorithm G-INS-i) and edited and trimmed by eye to remove short, ambiguously aligned regions (see Supplementary Files S1,S2). Edited alignments were used to generate the phylogenetic trees shown Katoh et al. (2002). Phylogenetic trees were generated from the resulting multiple sequence alignments (MSAs) using PhyML with subtree pruning and regrafting $(\mathrm{SPR})+$ nearest neighbor interchange $(\mathrm{NNI})$ moves and $\mathrm{X}^{2}$-like approximate likelihood ratio test (aLRT) clade support values, which serve as confidence scores much like bootstrap scores. Clades with aLRT scores $>0.95$ were deemed to have strong phylogenetic support (Anisimova and Gascuel, 2006; Guindon et al., 2009). Specific model parameters are provided in the figure legend for each PhyML analysis 
presented, however several additional MSAs and evolutionary models and parameters were tested for agreement with conclusions presented here (data not shown). Clades and evolutionary relationships mentioned in the text appeared consistently in independent phylogenetic analyses with different model parameters and MSAs.

\section{CLONING AND SEQUENCING OF CBLS AND CIPK FROM THE MOSS PHYSCOMITRELLA}

In order to verify expression and expected splice patterns of CBLs and CIPKs in an early-diverging land plant, we cloned CBLs and CIPKs identified from the model moss Physcomitrella. RNA was extracted from protonema and gametophores of Physcomitrella patens ssp patens ecotype Gransden 2004 using a CTAB/chloroform method similar to the one described by Chang et al. (1993). The RNA was reverse transcribed to produce cDNA using Superscript III Reverse Transcriptase (Invitrogen). Primers containing Invitrogen Gateway attB1 (forward primers) and attB2 (reverse primers) recombination sites were designed to amplify the coding sequences (CDSs) of each Physcomitrella CBL $(P p C B L)$ and CIPK (PpCIPK) genes (see Supplementary Table $\mathbf{S 3}$ for oligonucleotide sequences used in this study). CBL and CIPK transcripts were amplified using Phusion DNA Polymerase (Thermo-Fisher Scientific) following recommended manufacturer protocols on a MJ Research PTC100 or PTC-200 model thermocycler. PCR products were visualized on a $0.8 \%$ agarose gel, and products of the expected sizes were extracted using a QIAquick gel extraction kit (Qiagen) and cloned into the $\mathrm{pDONR}{ }^{\mathrm{TM}} /$ Zeo vector (Invitrogen) by Gateway $\mathrm{BP}$ reaction, following manufacturer recommendations. Samples from three or more clones for each gene were submitted to Elim Biopharmaceuticals, Inc. (Hayward, CA) for DNA sequencing.

\section{YEAST TWO-HYBRID ASSAYS}

In order to verify physical interactions among CBLs and CIPKs in a non-angiosperm plant, we cloned the CDS of each full-length $C B L$ and $C I P K$ transcript identified in Physcomitrella and tested interactions among PpCBLs and PpCIPKs in yeast two-hybrid (Y2H) assays using the yeast strain AH109 (Clontech Inc.). This strain is auxotrophic for leucine, tryptophan, histidine, and adenine. The CDSs of PpCBLs and PpCIPKs were cloned by Gateway $L R$ reaction into yeast two-hybrid gateway-compatible vectors (pGBT9-BS-GW and pGAD-GH-GW) derived from pGBT9-BS and pGAD-GH (Clontech). These vectors were transformed into yeast cells using the G-Biosciences FastYeast Transformation Kit and used to express CBL and CIPK fusions to the DNA-binding domain (BD) and activation domain (AD) of a split transcription factor. We screened CBL-BD fusions (pGBT9-BS-GW constructs) for interactions with CIPK-AD fusion proteins (pGAD-GH-GW constructs) and performed reciprocal screens among CIPK-BD and $\mathrm{CBL}-\mathrm{AD}$ fusion proteins to verify that the interactions were not vector-dependent. As negative controls, we verified that CBL-BD or CIPK-BD fusion proteins did not interact with the pGAD-GH empty vector (EV).

To perform Y2H screens, co-transformed cells were cultured to mid-log phase in MP Biomedical drop out base (DOB) liquid media lacking leucine and tryptophan (-LT), to ensure retention of vectors containing bait and prey constructs. We then adjusted the cultures to $\mathrm{OD}_{600}=0.05$ and divided them into four 10 -fold dilutions $\left(\mathrm{OD}_{600}=5 \times 10^{-2}, 5 \times 10^{-3}, 5 \times 10^{-4}, 5 \times 10^{-5}\right)$. $6 \mu \mathrm{l}$ droplets of each dilution were plated on agar DOB media (1) lacking leucine and tryptophan (-LT) to serve as a positive control for transformation and loading, (2) lacking leucine, tryptophan, and histidine (-LTH) to test for protein-protein interactions under low stringency, and (3) lacking leucine, tryptophan, histidine, and adenine (-LTHA) to test for interactions under stringent conditions. Cell growth was recorded at $48 \mathrm{~h}$ intervals over the course of 6 days.

\section{RESULTS AND DISCUSSION CBL-CIPK NETWORK COMPOSITION IN GREEN ALGAE, MOSS, AND OTHER LAND PLANTS}

CBLs and CIPKs have been previously identified among various land plants and chlorophyte green algae, though other chlorophytes appear to lack CBL-CIPK homologs (Weinl and Kudla, 2009). Utilizing recently available transcriptome data, we identified CBL and CIPK homologs from several charophyte green algae species: Coleochaete orbicularis, Klebsormidium flaccidum, Chaetospheridium globosum, Penium margaritaceum, and Chlorokybus atmophyticus. Interestingly, we identified a single CBL and single CIPK in each of these lineages, with one exception. We could not confidently identify a CIPK homolog from Chlorokybus, though this may due to incomplete transcriptome coverage. Additional CBL or CIPK homologs may be present in these taxa but undetected due to incomplete sequencing coverage, or additional homologs may simply not be transcribed at sufficient levels under sampled growth conditions. In agreement with our current understanding of evolutionary relationships among these organisms, charophyte green algae sequences display greater sequence similarity to land plant CBLs and CIPKs than chlorophyte homologs. Although there is no currently available genome sequence for any charophyte, only a single CBL and single CIPK were identified in the complete genome sequence of the chlorophytes Ostreococcus lucimarinus and Bathycoccus prasinos, consistent with prior findings (Weinl and Kudla, 2009). Though it is difficult to make genomic inferences about any charophyte green alga without an available complete genome sequence, our analyses suggest that green algae commonly contain a single CBLCIPK pair and that the CBL-CIPK network likely predates the split of chlorophyte and charophyte algae.

All CBLs and CIPKs analyzed in this study, including the most divergent homologs identified in algae, show strong conservation of domain architecture and important motifs. At approximately 200 amino acids (AAs) in length, CBLs contain one of a few variations of a localization at their N-termini, followed by 4 calcium-binding EF hand domains. The first EF-hand of CBLs is distinctive in that the calcium-binding loop is comprised of 14 rather than 12 AAs, however evidence suggests that it indeed binds calcium ions (Nagae et al., 2003). Identified full-length CIPKs are approximately 475 AAs in length and have a conserved domain architecture comprised of a N-terminal kinase domain and a $\mathrm{C}$-terminal autoinhibitory region with a diagnostic NAF domain that mediates interactions with CBLs. One previously identified CIPK from the chlorophyte green alga Chlorella 
(UniProt: C4P7Q5) differs, however, in that it possesses $2 \mathrm{NAF}$ domains in its C-terminus, though the significance and accuracy of the published domain architecture is unknown. Our homology search results corroborate the assertion that CBLs and CIPKs are not found in certain chlorophyte green algae, including the models Chlamydomonas and Volvox (Weinl and Kudla, 2009; Batistic et al., 2011). This pattern parallels trends in calcium channel evolution. The Chlamydomonas genome encodes several voltage-dependent calcium channels (VDCCs) and transient receptor potential (TRP) channels, which play critical roles in environmental sensing in metazoans, whereas sequenced land plant genomes do not contain discernible homologs from either family (Wheeler and Brownlee, 2008; Verret et al., 2010). Like most metazoans, Chlamydomonas is motile and, in addition to performing photosynthesis, readily grows heterotrophically. Chlamydomonas cells contain an organelle unlike any found in plants, the eyespot, that is involved in the calcium-mediated process of phototaxis (Witman, 1993). Based on these observations, it appears that some components of the calcium signaling machinery of certain chlorophyte green algae, such as Chlamydomonas, more closely resemble animal signaling networks in some aspects than those of land plants.

Taking advantage of the published genome sequence of the moss Physcomitrella patens, we determined the genomic complement of CBLs and CIPKs in this early-diverging model plant. We identified a total $4 \mathrm{CBL}$ and 7 CIPK predicted protein sequences in Physcomitrella, consistent with prior reports (Batistic and Kudla, 2009; Weinl and Kudla, 2009). One pair of CBLs (PpCBL2+3) and three pairs of CIPKs $(P p C I P K 1+5,3+4$, and $6+7)$ showed strikingly high sequence similarities at both the amino acid (73-93\% pairwise identity) and genomic level (42-52\% pairwise identity). Because of this observation and the inferred whole genome duplication (WGD) estimated to have occurred $\sim 45$ million years ago in Physcomitrella (Rensing et al., 2007), we hypothesized that pairs of CBLs and CIPKs are products of the recent WGD and that the "unpaired" CBLs (PpCBL1 and PpCBL4) and CIPK (PpCIPK2) may similarly possess cognate loci in the Physcomitrella genome. Consistent with this hypothesis, we identified paired loci for each gene and provisionally named these PpCBL5, PpCBL6, and PpCIPK8 (Figure 1). Although these loci showed relatively low percentage identity to their cognate loci compared to previously detected $C B L s$ and $C I P K s$, gene predictions using Augustus (Stanke et al., 2004) suggested these loci may encode partial or full-length proteins. Using RT-PCR, we amplified and cloned transcripts from $P p C B L 5$ and $P p C I P K 8$, however we failed to amplify transcripts from the $P p C B L 6$ locus using several primer pairs validated on genomic DNA (data not shown), despite testing cDNA from different developmental stages (protonema, gametophores, sporophytes). Pairwise alignment of the PpCBL4 and $P p C B L 6$ loci revealed a relatively low percentage identity, particularly in $P p C B L 4$ exonic regions, compared to other "sister" pairs of CBLs and CIPKs; these observations suggest that PpCBL6 may be a pseudogene. Sequenced $P p C B L 5$ and $P p C I P K 8$ transcripts detected from both gametophyte and sporophyte cDNA were found to contain premature termination cassettes (PTCs) in their spliced forms (see Figure 1, Supplementary File S3), which suggests that these transcripts may not be translated, at least under conditions that we tested. Physcomitrella CIPK8 contains a single nucleotide repeat (SNR), which are known to promote mutations and quickly change in length (Ellegren, 2004), spanning 31 bases in the retained PTC in cloned transcripts, further marking it as an unusual CIPK. Interestingly, $P p C B L 5$ and $P p C I P K 8$ show obviously stronger conservation in exonic regions (i.e., regions retained in spliced transcripts and that align to their sister gene's CDS) than intronic or regulatory regions (i.e., promoter and terminator). While these aberrant CBL and CIPK loci may simply be in early stages of pseudogenization, the unexpected finding that these loci are transcribed and spliced warrants further investigation into possible functions and may point toward a role for these transcripts as regulatory RNAs, as shown previously in animals (Korneev et al., 1999; Hirotsune et al., 2003; Tam et al., 2008). Like in Physcomitrella, expansion of the CBL-CIPK network in Arabidopsis, previously attributed to segmental duplications (Kolukisaoglu et al., 2004), can be traced to known WGD events in light of our current understanding of plant genome evolution (Cui et al., 2006). Independent expansion of other gene families in moss and angiosperms has been described, and this can obfuscate direct comparison and functional prediction of genes in widely divergent plants (Cui et al., 2006; Bowman et al., 2007; Rensing et al., 2008; Jiao et al., 2011).

\section{PHYLOGENOMIC ANALYSIS OF CBL REVEALS CONSERVATION OF THREE UNIQUE N-TERMINAL MOTIFS}

Phylogenomic methods extend the ability to determine relationships among distant homologs, facilitate functional prediction, and provide a framework for discovery of key features by identifying conserved regions of proteins (Eisen and Wu, 2002; Sjölander, 2004). Using maximum likelihood (ML) methods, we reconstructed the phylogeny of the CBL family in green lineages. Consistent with our hypothesis that land plant CBLs and CIPKs expanded from a simple module present in their common ancestor with algae, green algal CBLs cluster closely to one another with high confidence scores in phylogenetic analyses. Although algal CBLs do not consistently cluster with any particular clade of CBLs from land plants, they commonly show moderate phylogenetic affinity for a clade containing Arabidopsis CBL1 and CBL9 (Figure 2; see Supplementary Figure 1 for full tree), which play important roles in potassium nutrition through regulation of the AKT1 channel. Like Arabidopsis CBL1 and CBL9, green algal CBLs feature the dual-lipid modification motif MGCXXS/T or obvious relicts of this motif. Due to the retention of this motif among many embryophyte and green algal CBLs and the results of our phylogenetic analysis, we hypothesize that the dual-lipid modification motif is the ancestral localization mechanism of CBLs. This hypothesis is strengthened by the observation that distantly homologous neuronal calcium sensor (NCS) proteins feature a similar N-terminal motif (MGXXXS) that lacks the conserved cysteine residue but does trigger $\mathrm{N}$-myristoylation of the conserved glycine residue (Li et al., 2011). We designate homologs with the dual lipid modification motif as Type I CBLs (Figure 3 top). Consistent with the hypothesis that ancestral CBLs most closely resembled modern Type I homologs and gave rise to other types of CBLs, Type I CBLs are paraphyletic with respect to other CBLs. Arabidopsis CBLs containing the Type I dual lipid 


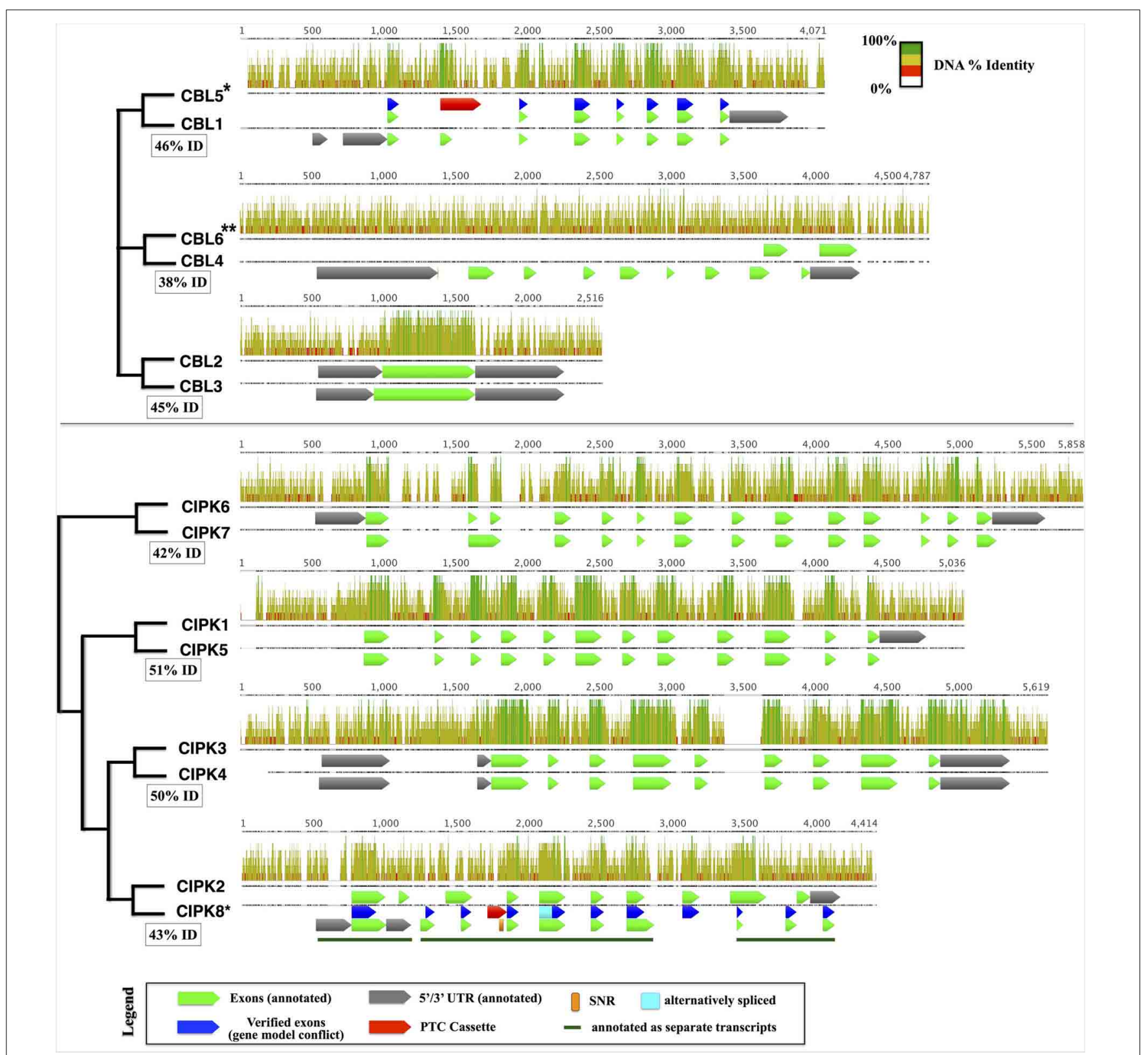

FIGURE 1 | Pairs of cognate CBLs (top) and CIPKs (bottom) in the Physcomitrella genome aligned using MAFFT. Displayed pairs of CBLS and CIPKs are genomic loci that are reciprocal best BLASTn hits within the genome, and inferred phylogenetic relationships are indicated by cladograms and described in the main text. Pairwise percentage nucleotide (nt) identity for pairs of genomic loci are displayed in boxes. Aligned nucleotides are displayed as bars shaded proportionally to percentage identity, and gapped regions in the alignment are represented by lines. Bar graphs indicate percentage identity (sliding window $=6 \mathrm{nt}$ ). Genes with cloned transcripts that do not encode full-length proteins under tested conditions are indicated with an asterisk $\left(^{*}\right)$ and genes lacking detectable transcripts are marked with two asterisks $\left.{ }^{* *}\right)$. In cases where our experimentally inferred gene model did not match the annotation, verified exons (blue), alternatively spliced regions (cyan), and premature termination cassettes (PTCs; red) are shown for comparison. CIPK8, which was annotated incorrectly as three separate transcripts, contains a long single nucleotide repeat (SNR) comprised of 31 thymidine (T) residues and described further in the main text. Sequences and associated annotations were extracted from the Physcomitrella genome v1.6 starting from 500 nucleotides (nt) upstream of the annotated $5^{\prime}$ UTR (750 nt upstream CDS for genes lacking $5^{\prime}$ UTR annotations) to $250 \mathrm{nt}$ downstream of the annotated $5^{\prime}$ UTR ( $500 \mathrm{nt}$ downstream the CDS) were extracted. Pairwise loci were aligned using MAFFT. Although PpCBL6 has annotated exons, there is no experimental evidence that any part of this locus in transcribed. modification motif have been shown to localize to the plasma membrane (D'Angelo et al., 2006; Cheong et al., 2007; Batistic et al., 2008). Mutational analyses using FP-fusions indicate that both $\mathrm{N}$-myristoylation and $\mathrm{S}$-acylation are required to target proteins to the plasma membrane, whereas either modification on its own results in endomembrane localization (Batistic et al., 2008). Although subcellular localization has not been investigated in early diverging plants or green algae, we speculate that the 


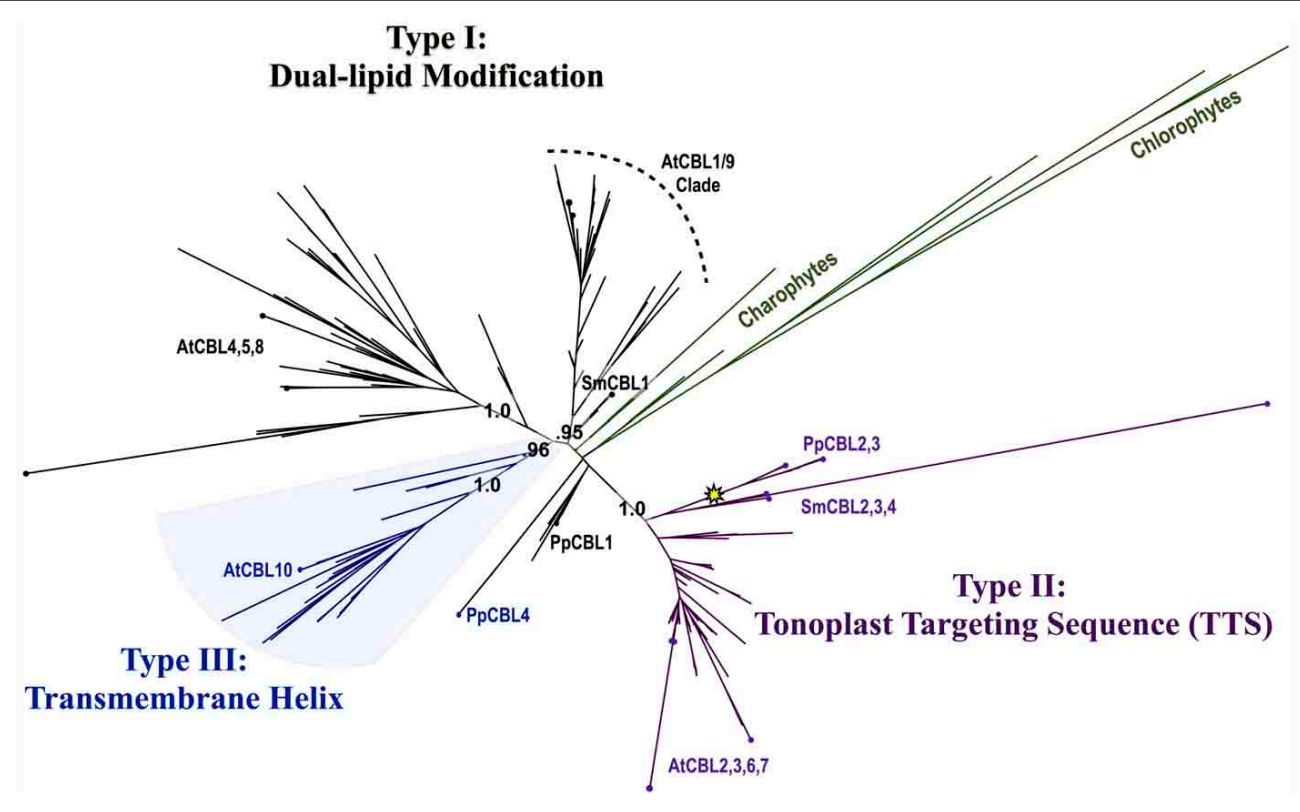

FIGURE 2 | Maximum Likelihood (ML) phylogenetic tree derived from multiple sequence alignment (MSA) of all CBL amino acid (AA) sequences analyzed in this study. Chlorophyte and charophyte green algal $\mathrm{CBL}$ and CIPKs are highlighted in green, and clades containing Type I-III
CBLs identified in this study are annotated. The yellow star indicates an inferred intron loss event. See Supplementary Table S1 for list of CBL sequences used in this study, Supplementary File S1 for MSA, and Supplementary File S2 for full phylogenetic tree. ancestral CBL-CIPK module may have participated in the regulation of integral membrane proteins at the plasma membrane, given the observed evolutionary trends and our understanding of CBL-CIPK function and biochemistry in Arabidopsis.

Phylogenetic analyses revealed a strongly supported (aLRT $=$ 1.0) clade that contains Physcomitrella CBL2 and CBL3 and Arabidopsis CBL2, CBL3, CBL6, and CBL7 (Figure 4). Physcomitrella CBL2 and CBL3 encode proteins that are $76 \%$ identical, and both genes lack introns, unlike other $C B L s$ from Arabidopsis or Physcomitrella. The clade also contains homologs from other non-angiosperms, including three CBLs from the lycophyte Selaginella moellendorffii. Like Arabidopsis, Selaginella CBLs in this clade contain multiple introns and exhibit a conserved exon-intron structure (data not shown), leading us to infer there was a likely reverse transcription event in the Physcomitrella lineage not shared with the lineage leading to lycophytes and angiosperms. Experimental work is needed to determine functional consequences of intron loss in Physcomitrella $C B L 2$ and $C B L 3$, however the effects and mechanisms of reverse transcription-mediated intron loss events and other means of intron loss are discussed elsewhere (Jeffares et al., 2006; Filichkin et al., 2010). Based on high sequence similarity, shared intron loss, and strong phylogenetic evidence, we infer that $P p C B L 2$ and $P p C B L 3$ are products of a lineage-specific gene duplication, likely the results of a recent WGD (Rensing et al., 2007, 2013). Both genes are orthologous to the four Arabidopsis CBLs contained in this clade. Arabidopsis CBL2 and CBL3 are also recent duplicates, as evidenced by their phylogenetic placement and very high sequence similarity ( $~ 92 \%$ AA identity) throughout their entire lengths. $C B L 3$ and $C B L 7$ are tandem duplicates, although
CBL7 is disparate from other Arabidopsis CBLs and contains a deletion in its $\mathrm{N}$-terminus between a degenerate dual-lipid modification motif and its first EF hand (Batistič and Kudla, 2009). Arabidopsis CBL6, which features an unusual first EF hand relative to other CBLs, is more distantly related to the three other AtCBLs in this clade and forms a clade with orthologous CBLs from other eudicots.

Arabidopsis CBL2, CBL3, and CBL6 have been reported to localize to the tonoplast (Batistič and Kudla, 2009). In the case of CBL2 and CBL3, it has been rigorously shown that an Nterminal motif known as the tonoplast targeting sequence (TTS) mediates its subcellular localization (Tang et al., 2012). The TTSs of Arabidopsis CBL2 and CBL3, with the consensus motif MSQCXDGXKHXCXSXXXCF, span 19 AA; and the last three positions of the motif overlap with positions $2-4$ in the dual lipid modification motif of Type I CBLs (i.e., MGCXXS/T), sharing a conserved cysteine residue found in all CBLs analyzed (see Figure 3, Supplementary File S1). This 19-AA fragment from either Arabidopsis CBL2 or CBL3 is necessary and sufficient for targeting of FP fusions to the tonoplast in Arabidopsis mesophyll cells (Tang et al., 2012), and strong sequence similarity suggests that CBL6 shares this targeting mechanism (Figure 3 middle). CBL7 is reported to show a diffuse nuclear and cytosolic localization based on the analysis of fluorescent fusion proteins (Batistic et al., 2008), however we are unaware of any rigorous attempts to determine its subcellular localization. Therefore, it appears that tonoplast localization is a generally conserved feature among angiosperm CBLs in this clade. We identified a TTS-like motif in all three Selaginella CBLs in this clade and in PpCBL3. Unlike PpCBL3, PpCBL2 does not contain an extended N-terminus and 


\begin{tabular}{|c|c|}
\hline $\begin{array}{c}\text { Type I: } \\
\text { Dual lipid modification } \\
\text { motif }\end{array}$ & 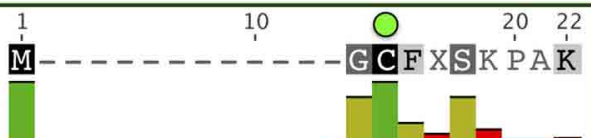 \\
\hline $\begin{array}{l}\text { 1. Ostreococcus tauri CBL1 } \\
\text { 2. Ostreococcus lucimarinus CBL1 } \\
\text { 3. Chlorella sp. CBL1 } \\
\text { 4. Penium margaritaceum CBL1 } \\
\text { 5. Coleochaete orbicularis CBL1 } \\
\text { 6. Chaetospheridium globosum CBL1 } \\
\text { 7. Klebsormidium flaccidum CBL1 } \\
\text { 8. Chlorokybus atmophyticus CBL1 } \\
\text { 9. Physcomitrella patens CBL1 } \\
\text { 10. Selaginella moellendorffii CBL1 } \\
\text { 11. Arabidopsis thaliana CBL1 } \\
\text { 12. Arabidopsis thaliana CBL4 } \\
\text { 13. Arabidopsis thaliana CBL5 } \\
\text { 14. Arabidopsis thaliana CBL9 }\end{array}$ & 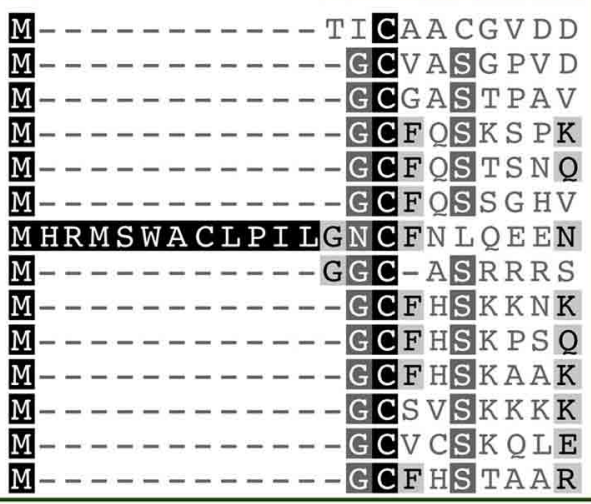 \\
\hline
\end{tabular}

\begin{tabular}{|c|c|c|}
\hline $\begin{array}{l}\text { Type II: } \\
\text { Tonoplast targeting } \\
\text { sequence (TTS) }\end{array}$ & $\begin{array}{l}\text { Consensus } \\
\text { Similarity }\end{array}$ & 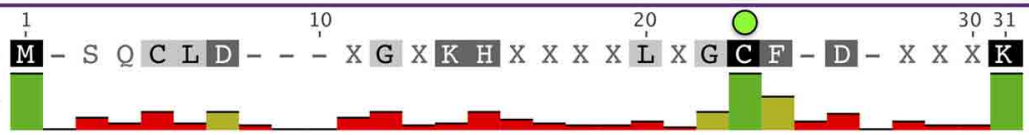 \\
\hline $\begin{array}{l}\text { 1. Physcomitrella patens } \\
\text { 2. Physcomitrella patens } \\
\text { 3. Selaginella moellendo } \\
\text { 4. Selaginella moellendo } \\
\text { 5. Selaginella moellendo } \\
\text { 6. Arabidopsis thaliana } \\
\text { 7. Arabidopsis thaliana } \\
\text { 8. Arabidopsis thaliana } \\
\text { 9. Arabidopsis thaliana }\end{array}$ & $\begin{array}{l}\text { CBL2 } \\
\text { CBL3 } \\
\text { ffii CBL2 } \\
\text { ffii CBL3 } \\
\text { ffii CBL4 } \\
\text { BL2 } \\
\text { BL3 } \\
\text { BL6 } \\
\text { BL7 }\end{array}$ & 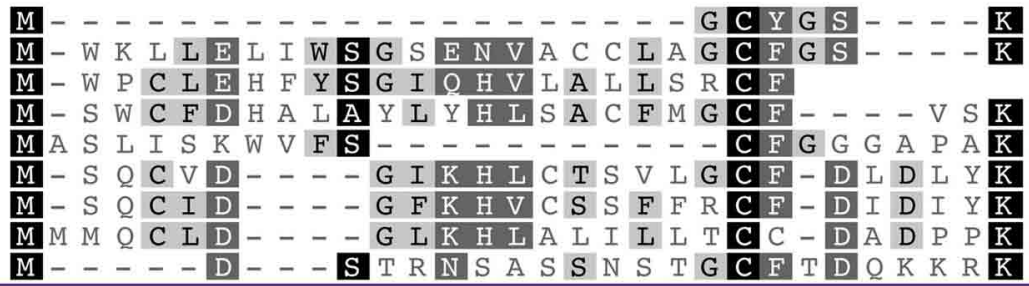 \\
\hline
\end{tabular}

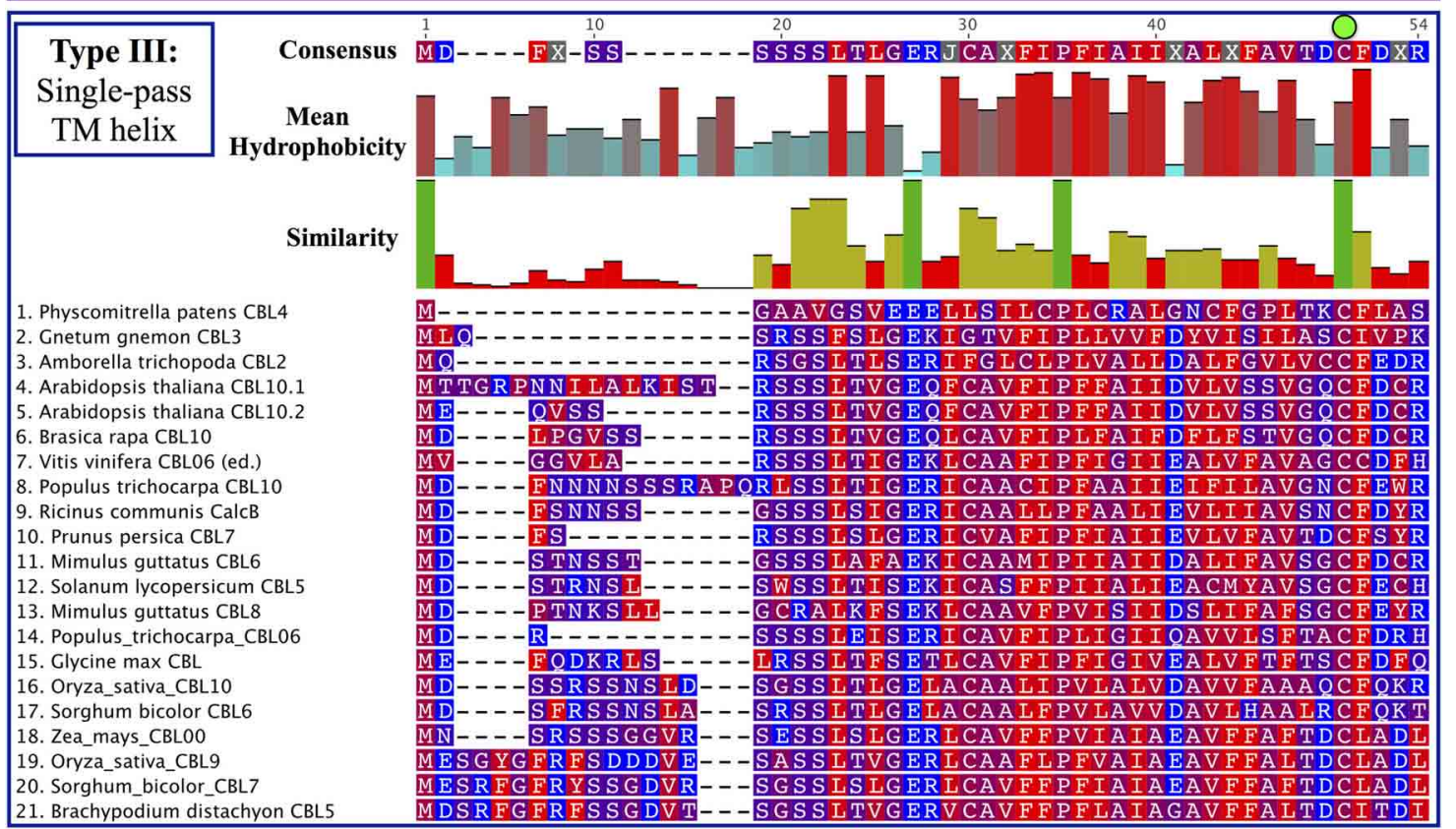

FIGURE 3 | CBL N-terminal localization motifs can be classified into three ancient types. Consensus sequences are provided above each MSA, and degree of conservation is indicated by bar graph and shading. Note the strictly conserved cysteine residues (green dots) in all three types of CBLs. (Top) Type I CBLs harbor a dual-lipid modification motif (MGCXXS/T) that triggers $\mathrm{N}$-myristoylation of the glycine residue and S-acylation of the cysteine residue. Most green algal CBLs identified to date are Type I CBLs or appear to retain signatures of the dual-lipid modification motif. (Middle) Type II CBLs are characterized by a N-terminal extension called the TTS that is found in nearly all CBLs contained in the Type II clade. Phylogenetic evidence suggests that PpCBL2 has lost its TTS through a mechanism such as gene conversion. (Bottom) Type III CBLs feature a long N-terminal extension that is predicted to constitute a transmembrane helix. Residues are colored according to hydrophobicity (red) or hydrophilicity (blue), and mean hydrophobicity and similarity are indicated by bar graphs. Although PpCBL4 does not cluster with seed plant CBLs that share a similar N-terminal extension, we propose that it is targeted in a similar manner to other Type III $\mathrm{CBLs}$ based on sequence analysis of its $\mathrm{N}$-terminal extension. 


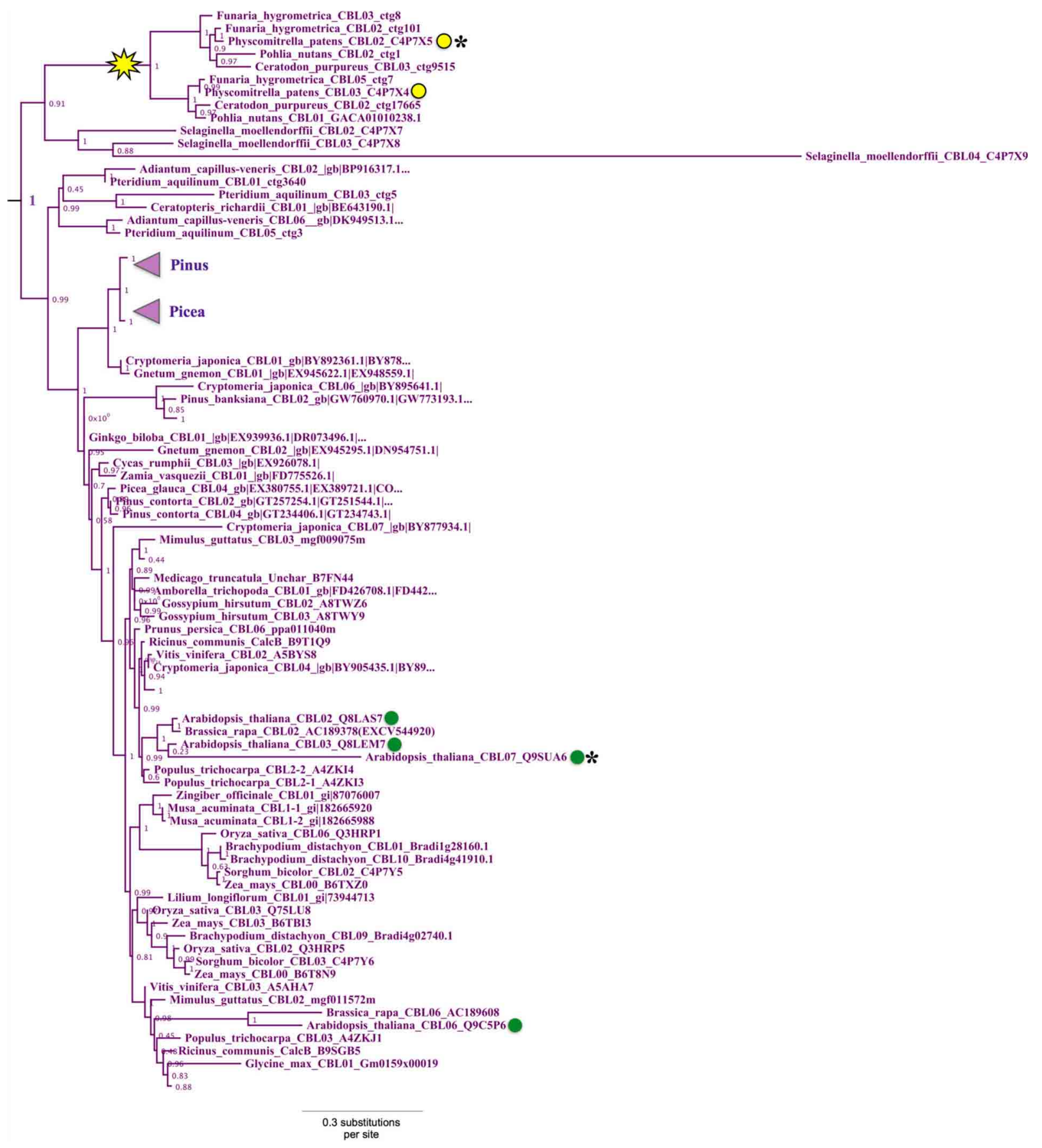

FIGURE 4 | Close-up of Type II CBL clade from ML tree shown in Figure 2. Confidence scores (aLRT) are shown for each clade, and the yellow star denotes an inferred intron loss event. Some clades are unlabeled or collapsed for clarity (see full tree in Supplementary File S2). Type II CBLs are distinguished by the presence of an $\mathrm{N}$-terminal tonoplast targeting sequence (TTS), however certain members (asterisks) of the Type II clade have lost or degenerate TTSs. Arabidopsis CBLs in this clade (green dots) contain the TTS or a degenerate form of it; whereas the two Physcomitrella CBLs (yellow dots) in this clade sharply differ in that CBL2 has a Type I dual-lipid modification motif and CBL3 has a TTS. Presence of the TTS in Selaginella homologs within the clade (see Figure 3 ) suggests that CBL2 has lost its TTS, through a mechanism such as gene conversion, for example. instead contains the Type I dual lipid modification motif. Given these trends, we posit that the TTS is a synapomorphy of this clade and that PpCBL2 lost its TTS via deletion or partial gene conversion, as described elsewhere (Jeffares et al., 2006). Based on strong phylogenetic support and TTS motif conservation, we designate homologs contained in this clade as Type II CBLs.

Phylogenetic analyses also identified a strongly supported clade that contains Arabidopsis CBL10, the only Arabidopsis CBL 


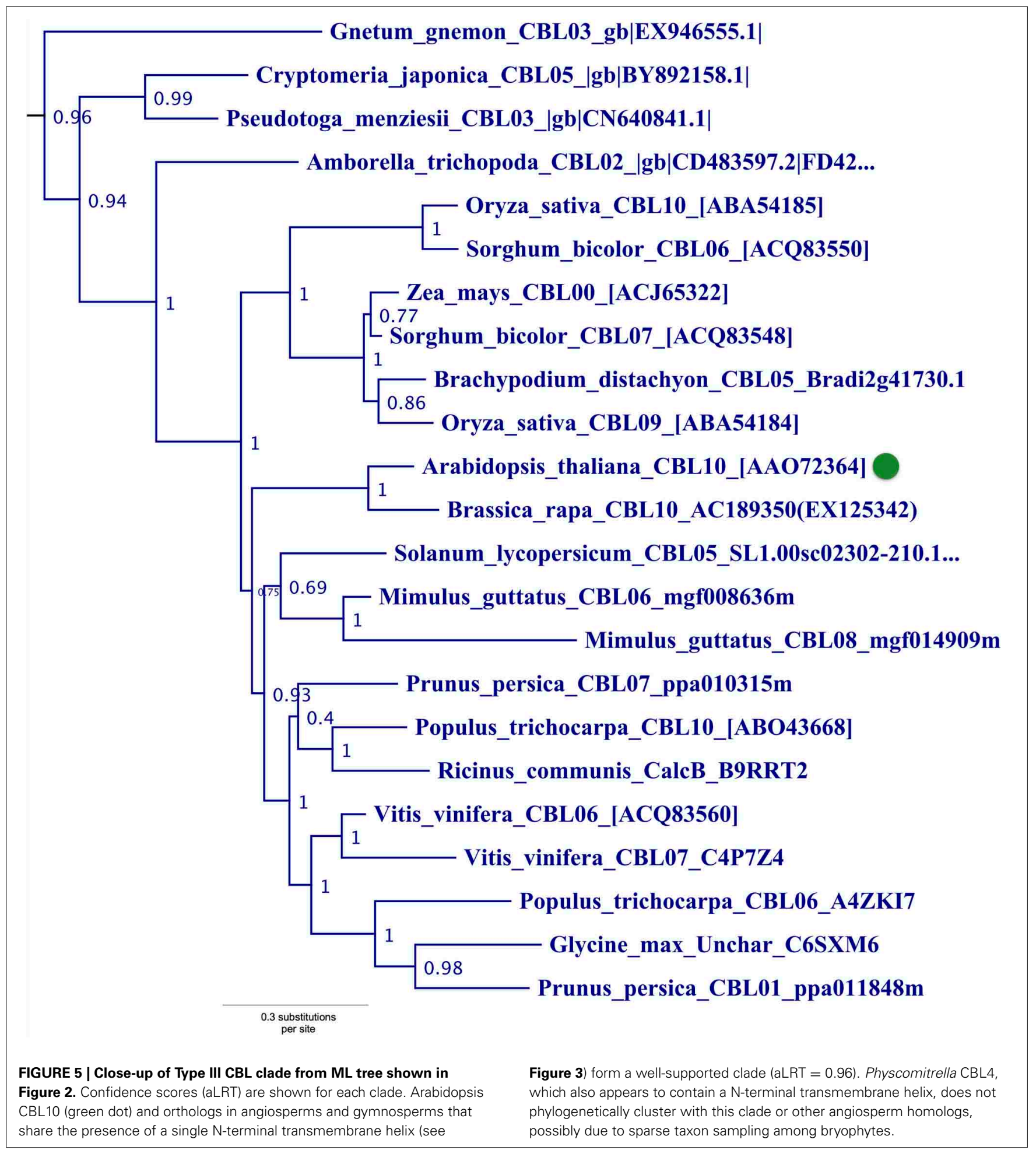

predicted to contain a transmembrane (TM) helix for membrane association (Figure 5). This clade contains orthologs from all studied angiosperms and gymnosperms, indicating this clade is conserved among seed plants; and all members of this clade with full-length sequences exhibit a predicted N-terminal transmembrane helix. Like members of the AtCBL10 clade, Physcomitrella
CBL4 contains an extended N-terminus, which we posited may form a transmembrane helix (Figure 3 bottom). Various TM topology prediction methods disagree on whether AtCBL10 or PpCBL4 contain a predicted TM helix (data not shown), however visual inspection of hydrophobicity and patterns of conservation in MSAs suggests that both AtCBL10 and PpCBL4 contain 


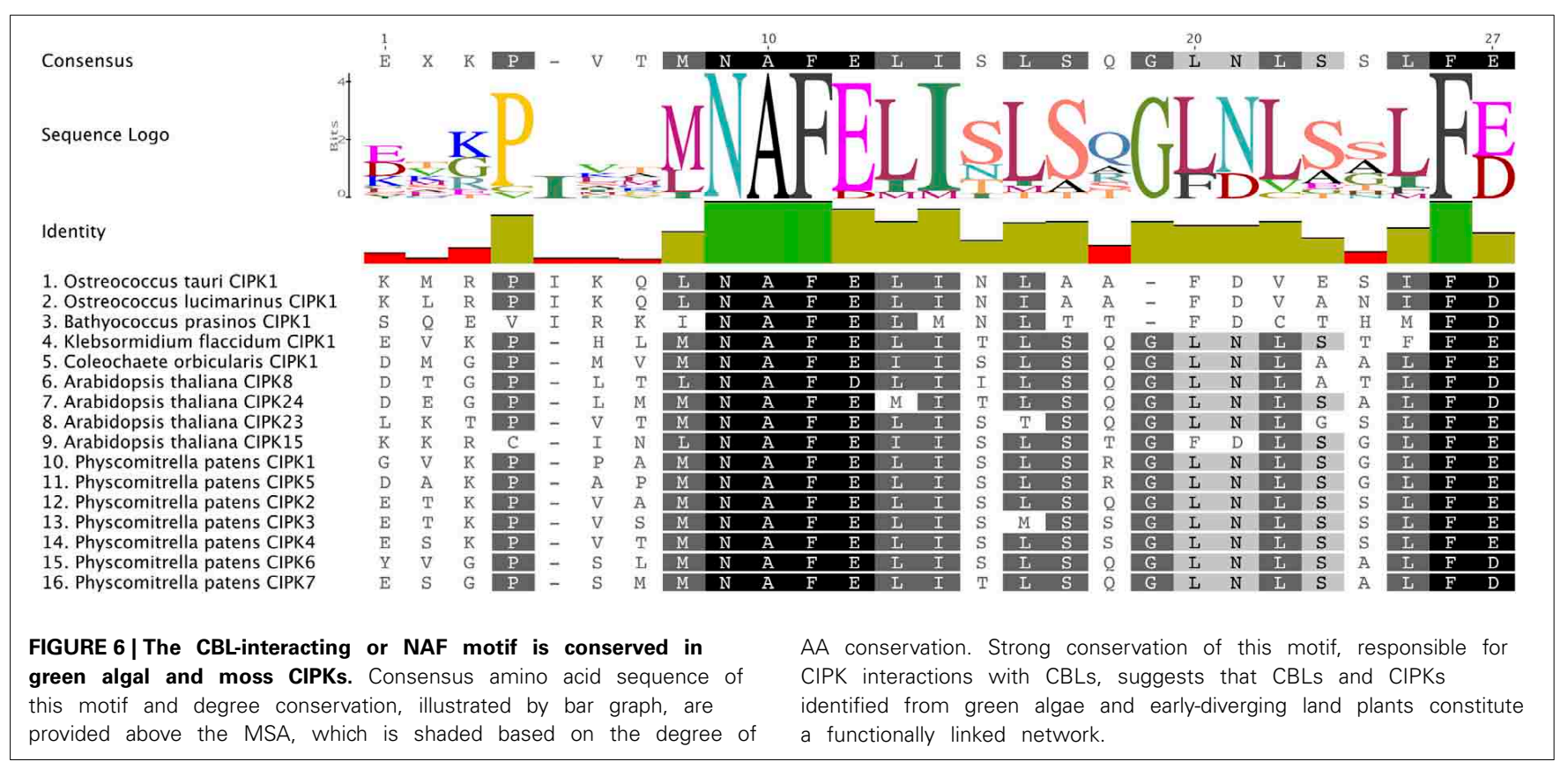

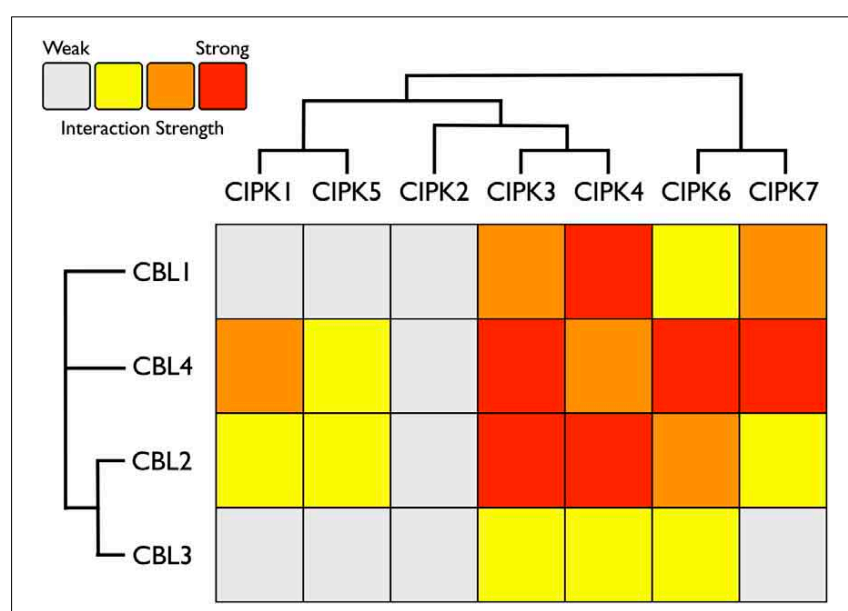

FIGURE 7 | Heat map summarizing yeast two-hybrid (Y2H) results for all Physcomitrella CBL and CIPK combinations. Each CBL and CIPK was fused to activation domain (AD) or DNA-binding domain (BD) of a split transcription factor and screened for interactions between CBL-AD/CIPK-BD fusion proteins and CBL-BD/CIPK-AD fusions. Interaction strength was inferred by serial growth dilutions on selective media lacking one or two auxotrophic markers and summarized qualitatively by heat map. Red boxes indicate vigorous growth on -LTHA plates (see Materials and Methods); orange boxes indicate weaker growth on -LTHA plates. Yellow boxes indicate robust growth on -LTH plates but no growth on -LTHA plates. Gray boxes indicate weak growth on -LTH plates, but each CBL-CIPK interaction conferred better growth than the empty vector (EV) control. Representative images of each assay are shown in Supplementary Figure S3. Inferred phylogenetic relationships of Physcomitrella CBLs and CIPKs are indicated by cladogram and described in the main text.

$\mathrm{N}$-terminal TM helices. The presence of this TM helix raises the possibility that PpCBL4 may be an AtCBL10 ortholog, however our phylogenetic data neither favor nor disfavor this hypothesis. More thorough coverage of sequence data from early-diverging plants is likely required to test this possibility and determine whether Type III CBLs are monophyletic or not. The Arabidopsis $C B L 10$ transcript is reportedly processed into mRNAs that encode proteins with two distinct $\mathrm{N}$-termini, though both share the same TM helical region. Alternative splicing is mediated by a unique 8th intron (other rice and Arabidopsis CBLs contain 6 or 7 introns) toward the $5^{\prime}$ pend of the transcript. Both Physcomitrella CBL4 and Arabidopsis CBL10 share a very similar exon-intron structure (data not shown), though we did not find evidence of alternative splicing in $P p C B L 4$.

The typically short length and strong structural conservation of EF hand proteins like CBLs can complicate phylogenetic reconstruction, as relatively few substitutions can significantly influence results. Due to biophysical constraints, EF hand domains typically exhibit strong sequence conservation at positions that coordinate calcium ion binding. However, variation seen among EF hands of CBLs are predicted to have widely differing affinities for calcium ions, thereby facilitating functional diversity at the level of calcium binding. The 4th EF hand (EF4) of Physcomitrella CBL4 is unusual in that it contains non-polar residues at two of the positions that coordinate calcium ion binding, rather than negatively charged residues as seen in virtually all other EF hands. Therefore, it appears likely that it does not bind calcium. Indeed, studies of other calcium signaling pathways have underscored the plasticity of signaling components during evolution. The model yeast Saccharomyces cerevisiae contains a single-copy gene encoding a calmodulin (CaM), a widely studied type of calcium sensor in eukaryotes. This gene, $C M D 1$, is indispensable for survival of the cell. Surprisingly, molecular genetic analysis suggests the CaM's ability to bind calcium ions is dispensable for its most vital functions, and its fourth EF hand is unable to bind calcium (Cyert, 2001). Plants contain a suite of typical CaMs and widely divergent CaM homologs, some of which either lack the ability to bind calcium ions or coordinate them in an unusual manner 
(McCormack and Braam, 2003). Further work is needed to clarify the capacity and affinity of identified CBLs for calcium binding, particularly among non-angiosperm CBLs.

There has been some debate as to the localization of Arabidopsis CBL10; various reports indicate localization to the tonoplast (Kim et al., 2007; Batistic et al., 2010) or plasma membrane (Quan et al., 2007; Ren et al., 2013). Although the multiple isoforms of CBL10 may account for different localization patterns, Arabidopsis CBL10 is most strongly expressed in shoots and is suggested to participate in the regulation of a NHX-family, $\mathrm{Na}^{+} / \mathrm{H}^{+}$exchanger believed to function in the sequestration of sodium ions within the vacuole. A model has emerged wherein CBL10 plays a regulatory role in the SOS pathway akin to that of CBL4/SOS3 (Kim et al., 2007; Tang et al., 2013). In root hair and cortical cells, the Type I Arabidopsis CBL4 forms a complex with CIPK24, and together they regulate the activity of the plasmalemma-localized $\mathrm{Na}^{+} / \mathrm{H}^{+}$exchanger SOS1 (syn. NHX7) and facilitate the extrusion of sodium ions from the plant. In shoot mesophyll cells, CBL10 complexes with CIPK24, and together they putatively regulate the activity of an unidentified tonoplast-localized $\mathrm{Na}^{+} / \mathrm{H}^{+}$exchanger and facilitate sequestration of sodium ions in the vacuole. A recent publication proposes a role for CBL10 in the regulation of the plasmalemma-localized potassium channel AKT1 (Ren et al., 2013), which has been rigorously shown to be subject to regulation by CBL1 and CBL9 acting in concert with CIPK23 (Li et al., 2006; Xu et al., 2006). Our phylogenetic results indicate that the single-pass $\mathrm{N}$-terminal TM helix is a synapomorphy of the AtCBL10 clade. Physcomitrella CBL4 likewise contains a N-terminal TM helix and may be orthologous, therefore we designated these homologs Type III CBLs.

Different membranes of the eukaryotic cell have distinct phospholipid profiles, which can serve as a basis for subcellular targeting. Moreover, each particular membrane is commonly composed of distinct microenvironments with unique lipid and protein populations. Together, proteins and lipids are thought to form functional modules in cellular membranes, with membrane-targeted kinases recognized as common regulatory modules (Engelman, 2005). For these reasons, we expect that CBL-CIPK complexes are likely targeted not only to specific membranes but to precise sites within membranes where they interact and function with molecular partners (Bhatnagar and Gordon, 1997; Levental et al., 2010). Elevation of free calcium in the cytosol is localized and transient, partly due to effects of $\mathrm{Ca}^{++}$-ATPases and $\mathrm{Ca}^{++} / \mathrm{H}^{+}$antiporters and proteins that act as buffers. Because calcium signatures occur locally, calcium sensors must operate in close proximity to the channels responsible for calcium elevation (Fogelson and Zucker, 1985; Gilroy et al., 1993; Roberts, 1994; Clapham, 2007). In light of this, we interpret the conservation of CBL localization motifs among distantly related plants as a likely consequence of constraints on CBL-CIPK subcellular localization.

Although several studies have examined CBL localization, it remains unclear whether CBLs display a predominantly static or dynamic localization at protein maturity. Our analyses demonstrate that the cysteine residue occupying the third position in the Type I motif (MGCXXS/T) is perfectly conserved among CBLs from widely divergent organisms and paralogous clades.
In Type I and Type II CBLs, this residue has been shown to be $\mathrm{S}$-acylated, and the modification is required for known protein functions (Batistic et al., 2008; Batistič, 2012; Tang et al., 2012). Based on its striking conservation, we predict that $\mathrm{S}$-acylation of this conserved residue is a shared among CBLs, at least under certain conditions. It is well established that S-acylation is a reversible post-translational modification and that it can strongly impact protein localization and can be critical for protein function (Bijlmakers and Marsh, 2003; Hemsley and Grierson, 2008). Prior research has pointed toward a role for S-acylation in finelevel targeting of proteins to specific membrane microenvironments (Bhatnagar and Gordon, 1997; Mumby, 1997; Dunphy and Linder, 1998; Levental et al., 2010). We predict that N-terminal Sacylation at this conserved residue functions, at least in part, as a mechanism for precise and dynamic localization of CBLs.

\section{CONSERVATION OF THE NAF MOTIF AND CBL-CIPK INTERACTIONS IN PHYSCOMITRELLA}

The CBL-CIPK network is mediated by a conserved CBLinteracting domain (also known as the NAF or FISL motif) in CIPKs. Our MSA of the CIPK family indicates that the NAF domain is strongly conserved, with many identical residues, among algal CIPKs and all CIPKs from Arabidopsis and Physcomitrella (Figure 6). This observation is consistent with our prediction that CBLs and CIPKs from green algae and early diverging embryophytes function together as a module. To confirm our presumption that Physcomitrella CBLs and CIPKs physically interact with each other and lend support to our interpretation of these protein families as functionally connected in early-diverging plants, we performed $\mathrm{Y} 2 \mathrm{H}$ screening and characterized physical interactions between full-length PpCBLs and PpCIPKs in yeast cells.

Consistent with our expectations, CBLs and CIPKs from Physcomitrella showed physical interactions in yeast cells. All combinations of PpCBL and PpCIPK fusion proteins showed physical interactions in yeast (Supplementary Figure S2), but specific CBL-CIPK combinations showed very strong interactions with select partners, consistent with the hypothesis that particular CBLs show preferential interactions with cognizant CIPKs (Figure 7). We observed that "creeter" CIPKs displayed overlapping, though not identical, interaction profiles with their most closely related homolog. CIPK1 and CIPK5 interact moderately with CBL2 and CBL4 and weakly with CBL1 and CBL3. CIPK3 and CIPK4 interact weakly with CBL3 but moderately to strongly with CBL1, 2, and 4. CIPK6 and CIPK7 interact strongly with CBL4 and weakly to moderately with CBL1, 2, and 3 . We observed only weak interactions between CIPK2, which lacks a "sister" CIPK, and any CBL, despite conservation of its NAF domain and phylogenetic proximity to the highly interactive CIPK3 and CIPK4.

Among the CBLs, CBL4 shows the highest number of strong connections to CIPKs, and it interacts very strongly with CIPK6 and CIPK7, members of the green algal clade of CIPKs. CBL1, a Type I CBL without clear phylogenetic affinities to angiosperm CBLs, most strongly interacts with CIPK4 and shows very weak interactions with CIPK1 and CIPK5. CBL3 shows clearly weaker interactions with CIPKs than its close paralog CBL2, although 


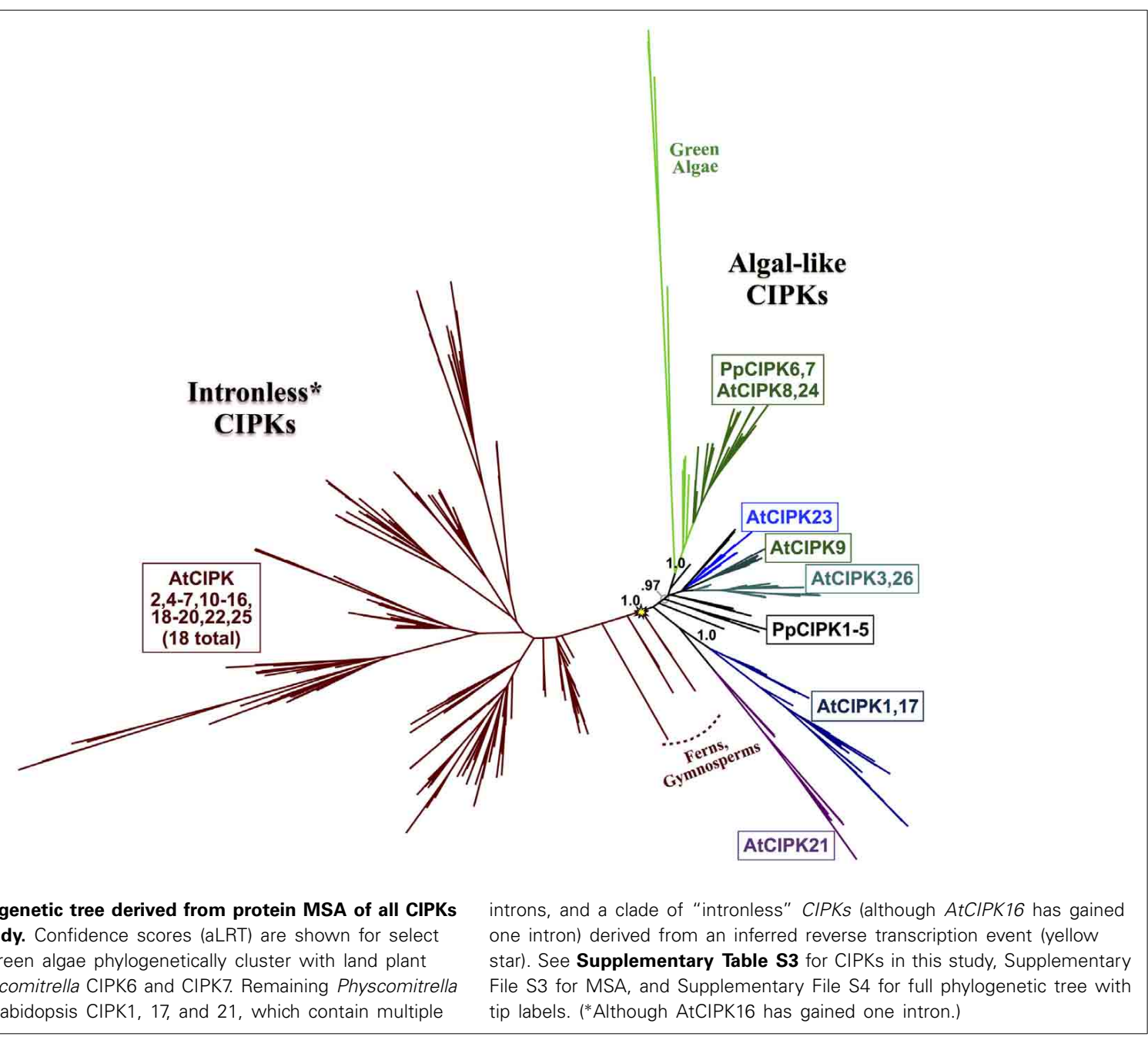

their interaction profiles are similar. CBL2 and CBL3 both interact most strongly with CIPK3, CIPK4, and CIPK6. These data support the model of a highly interconnected signaling network, however interaction patterns may differ significantly in moss cells due to differences in post-translational modifications, subcellular localization, expression, and other factors. Nonetheless, these results provide a guide for genetic analyses in moss and lend confidence to the interpretation that CBLs and CIPKs are functionally linked in early-diverging plants and constitute an ancient signaling network.

\section{PHYLOGENOMIC IDENTIFICATION OF THE ANCESTRAL OR “GREEN ALGAL-TYPE" CLADE OF CIPKs}

Phylogenomic analyses of the CIPK family were pursued, as described for CBLs, to decipher evolutionary patterns to facilitate identification of functionally meaningful groups, which would be expected to show conservation across diverse land plants. Our phylogenomic analyses of CIPKs (Figure 8; see Supplementary Figure S3 for full tree) indicated most Arabidopsis CIPKs (18 of 26) are contained within an "intronless" clade (although CIPK16 contains a single intron that is inferred to be from an introngain event), consistent with prior analyses by Kolukisaoglu et al.
(2004). We used conifer protein sequences from this clade as queries for tBLASTn searches of Picea chromosomal sequences (available at http://congenie.org) and did not identify introns in expected locations for intron-containing CIPKs (data not shown). Based on these observations, we posit that a reverse transcription event occurred before the split of gymnosperms and angiosperms and is a conserved feature of this clade. All Physcomitrella CIPKs contain multiple introns, and none cluster with the intronless clade. Physcomitrella CIPK1-CIPK5 share high sequence similarity (83-93\% pairwise); and in our analyses, they were placed with strong confidence in a clade with homologs from other mosses, indicating they are paralogs in respect to their closest seed plant homologs. This clade of moss homologs is likely orthologous ( $\mathrm{aLRT}=0.97$ ) to three clades of CIPKs conserved across seed plants: the aforementioned intronless clade, a clade containing AtCIPK21, and a clade containing AtCIPK1 and AtCIPK17.

Arabidopsis CIPK3+CIPK26, CIPK9, and CIPK23 each represent strongly supported (aLRT $=1.0$; see Supplementary Figure S3) clades that cluster with one another and contain homologs in fully sequenced angiosperm genomes and, at least for the CIPK3 + CIPK26 and CIPK23 clades, in gymnosperms. 
Whereas CIPK9 and CIPK23 regulate potassium transport and function in root and shoot tissues (Cheong et al., 2007; Pandey et al., 2007), CIPK3 has been implicated in abscisic acid (ABA)dependent regulation of seed germination (Pandey et al., 2008), therefore homologs from seed plants as distantly related as gymnosperms might conceivably have a conserved regulatory role in seeds, given their strong conservation.

Physcomitrella CIPK6 and CIPK7 belong to a clade that contains Arabidopsis CIPK8 and CIPK24 and, importantly, contains all green algal CIPKs identified (Figure 9) with high confidence
$(\mathrm{aLRT}=1.0)$. Although Physcomitrella and Arabidopsis each contain two homologs in this clade, Physcomitrella CIPK6 and CIPK7 (72\% AA pairwise identity) are the products of a gene duplication that occurred after the split between mosses and the lineage leading to vascular plants. In contrast, Arabidopsis CIPK8 and CIPK24 (60\% pairwise identity) each represent a separate, strongly supported clade with orthologs in other angiosperms, implying that they derive from duplications that occurred during seed plant (most likely angiosperm) diversification. Based on our results, we posit that Physcomitrella CIPK6 and CIPK7 and

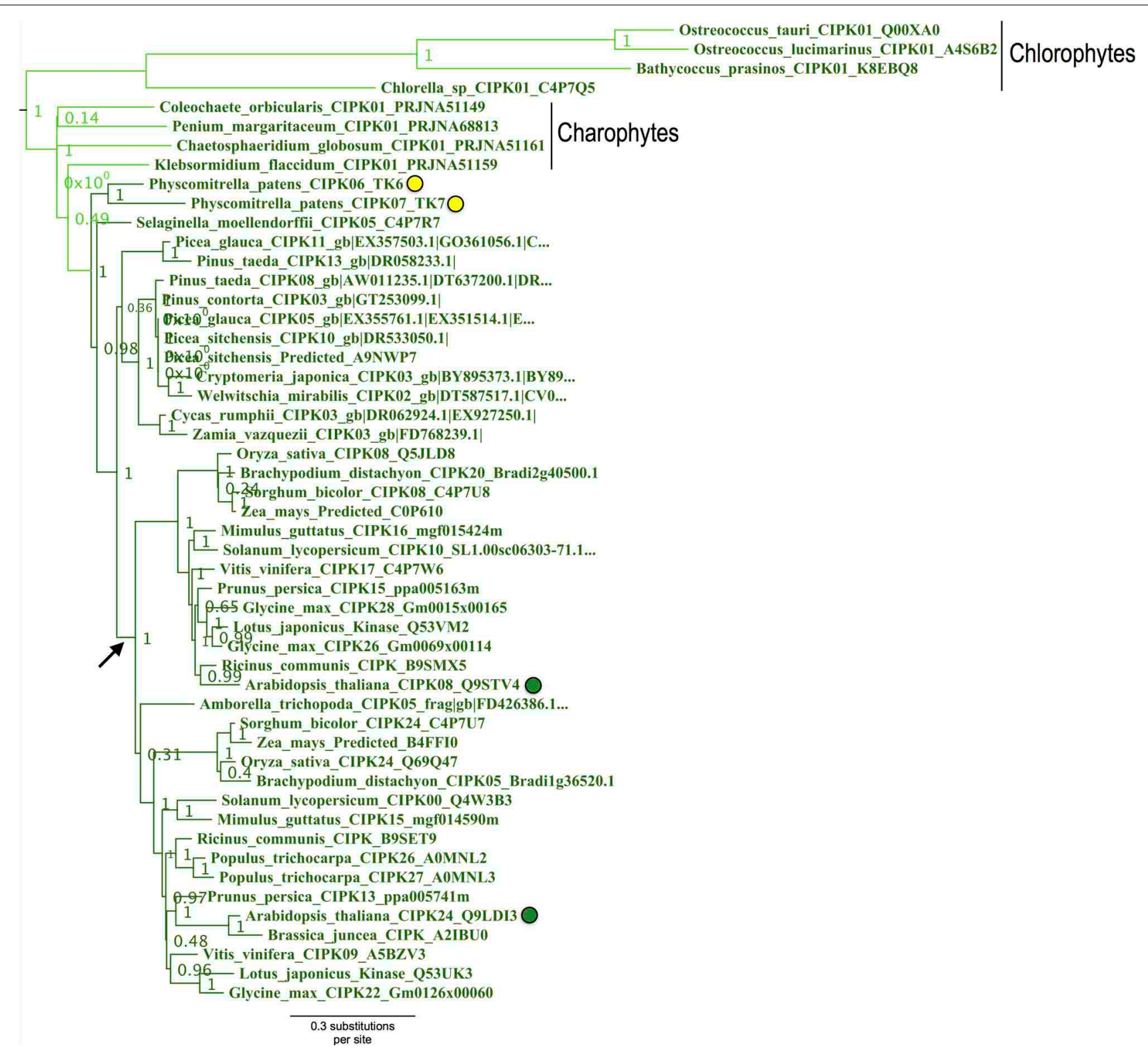

FIGURE 9 | Close-up of the "green algal-type" CIPK clade from ML tree shown in Figure 8. Confidence scores (aLRT) are shown for each clade. Phylogenetic evidence strongly supports the existence of a clade ( $a L R T=1.0$ ) containing all CIPK homologs identified from chlorophyte and charophyte green algae, as well as two CIPKs each from Physcomitrella (yellow dots) and Arabidopsis (green dots). Physcomitrella CIPK6 and CIPK7 are recent paralogs and sister to one another in our analyses. In contrast, Arabidopsis CIPK8 and CIPK24 each have clear orthologs in other sequenced angiosperms, and these clades appear to have arisen from a gene duplication that occurred around the time of divergence of angiosperms (arrow). 


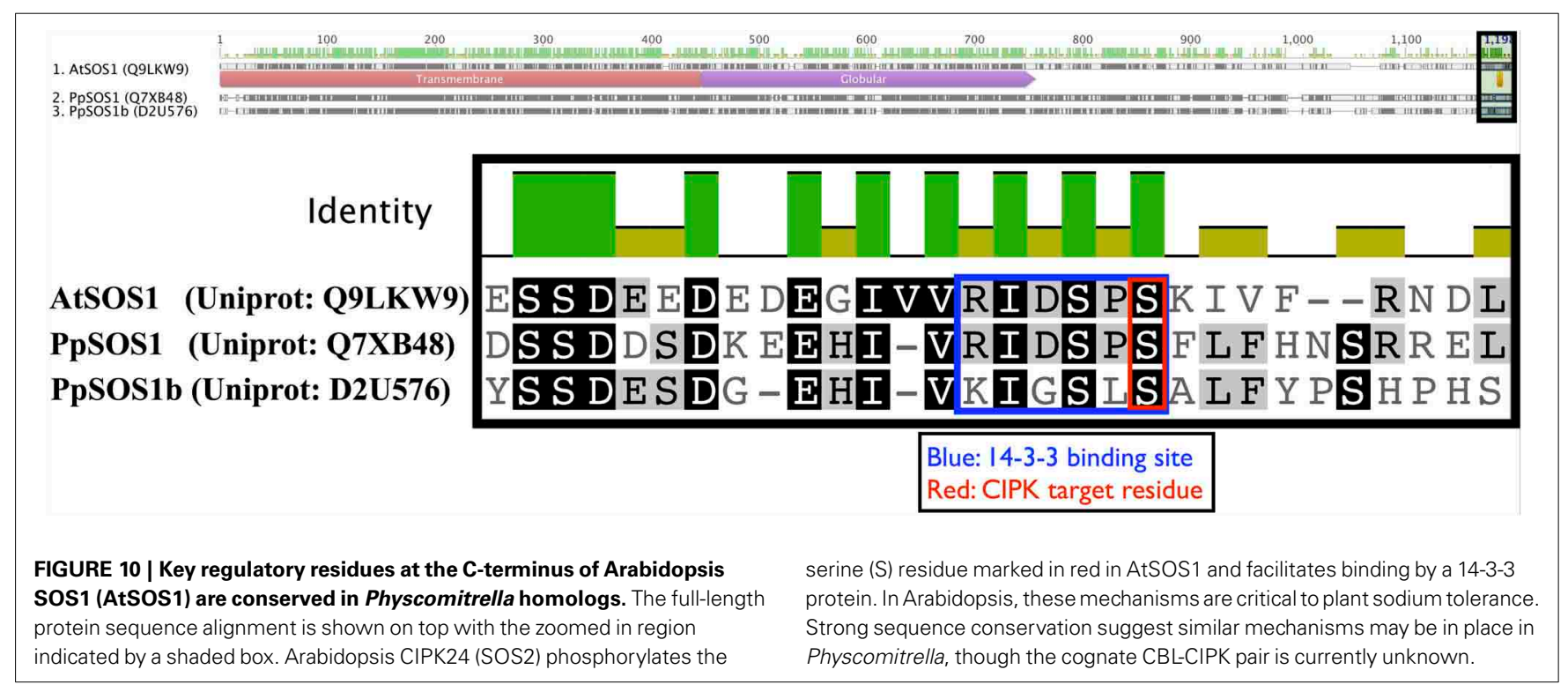

Arabidopsis CIPK8 and CIPK24 (SOS2) most closely resemble the ancestral or "green algal-type" CIPK and, due to their orthology, most likely to reflect ancestral function(s) of the CBL-CIPK network.

Arabidopsis CIPK8 is believed to be a positive regulator of the low-affinity phase of the primary nitrate response and has been implicated in glucose sensing, although mechanistic details are unknown at this time (Hu et al., 2009). Arabidopsis CIPK24, the first functionally characterized CIPK, plays a critical function in sodium tolerance through CBL4(SOS3)-modulated phosphorylation of the $\mathrm{Na}^{+} / \mathrm{H}^{+}$exchanger SOS1. There is substantial evidence that orthologs of CBL4 and CIPK24 in other flowering plant lineages have similar functions (Martínez-Atienza et al., 2007; Tang et al., 2010). Given the phylogenetic proximity of Arabidopsis CIPK24 to green algal CIPKs, future work will test whether green algal CIPKs, and Physcomitrella CIPK6 and CIPK7, function in $\mathrm{Na}^{+} / \mathrm{K}^{+}$homeostasis or possibly more broadly regulate ion transport. It has already been established that two orthologs of SOS1 in Physcomitrella (PpSOS1 and PpSOS1b) are required for proper $\mathrm{K}^{+} / \mathrm{Na}^{+}$ratios and sodium tolerance (Quintero et al., 2011). Interestingly, a 6 AA Cterminal motif of AtSOS1 that is a phosphorylation substrate of CIPK24 and a $14-3-3$ protein-binding site is $100 \%$ identical to PpSOS1 and 50\% identical to PpSOS1b, and the target serine is conserved in both homologs (Figure 10). Physcomitrella SOS1 has further been shown to confer enhanced $\mathrm{NaCl}$ tolerance when heterologously expressed in yeast, and the effect is strengthened by coexpression with Arabidopsis CBL4 and CIPK24 (Fraile-Escanciano et al., 2010). Collectively, these observations suggest that the SOS pathway is conserved across land plants and may be conserved among some green algal lineages. Functional molecular analyses of CBLs and CIPKs in early-diverging plant and algal lineages could provide core insights and clarify the increasingly complex picture of calciumregulated abiotic stress responses in Arabidopsis and agricultural species.

\section{CONCLUSIONS}

Prior publications (e.g., Batistič and Kudla, 2009; Weinl and Kudla, 2009) have mentioned the apparent expansion of the CBLCIPK network in terms of the total numbers of CBLs and CIPKs found in algae and early diverging plants compared to their angiosperm counterparts. Here, we present phylogenetic evidence that the CBL-CIPK network has expanded independently in multiple plant lineages, including mosses and angiosperms. It appears that the common ancestor of mosses and vascular plants likely contained three CBLs distinguishable by N-terminal localization motifs, which likely are synapomorphies among ancient CBL subfamilies. We have identified a clade of CIPKs containing all green algal homologs and two representatives from Physcomitrella and Arabidopsis. Phylogenetic analysis demonstrates that the Physcomitrella and Arabidopsis members of this clade are the products of independent gene duplications and the earliest land plants likely contained a single homolog from this clade. The concurrent pairing of CBLs and CIPKs in available genomes and transcriptomes, the striking conservation of the NAF domain, and our $\mathrm{Y} 2 \mathrm{H}$ results all point toward a physically and functionally connected CBL-CIPK network across plants and algae.

The function(s) of CBL-CIPK pairs found in green algae remains an open and intriguing question, and our identification of charophyte CBL-CIPK pairs expands the list of potential models for this inquiry. The conspicuous expansion of the network in several land plant lineages appears to have been driven largely by WGDs, and we hypothesize that duplicated members were adapted for novel signaling pathways and precise roles in particular cells and tissues. Research on molecular processes modulated by CBLs and CIPKs has intensified in recent years, and researchers are beginning to investigate CBL-CIPK functions in non-model angiosperm species. The field is prime for investigation of CBL-CIPK functions in earlier diverging land plants, and research in this area will enhance our understanding of the molecular evolutionary basis of the colonization of land by plants. 


\section{FUNDING}

This research is supported by a grant from the National Science Foundation (to Sheng Luan).

\section{ACKNOWLEDGMENTS}

We thank Dr. Stefan Rensing and Ryan Melnyk for helpful discussions on moss biology and evolutionary reconstruction of gene families and thank Dr. Peggy Lemaux for her mentorship and efforts to make this research possible. We are grateful to Dr. Ruth Timme for her assistance with the identification of charophyte CBLs and CIPKs. We gratefully acknowledge an NSF Graduate Research Fellowship Program fellowship to Thomas J. Kleist and a Sponsored Projects in Undergraduate Research fellowship and Biology Scholars Program awards to Andrew L. Spencley.

\section{SUPPLEMENTARY MATERIAL}

The Supplementary Material for this article can be found online at: http://www.frontiersin.org/journal/10.3389/fpls.2014.00187/ abstract

Supplementary Table S1 | CBL sequences used in this study.

\section{Supplementary Table S2 | CIPK sequences used in this study.}

\section{Supplementary Table S3 | Oligonucleotides used in this study.}

\section{REFERENCES}

Albrecht, V., Ritz, O., Linder, S., Harter, K., and Kudla, J. (2001). The NAF domain defines a novel protein-protein interaction module conserved in $\mathrm{Ca}^{2+}$-regulated kinases. EMBO J. 20, 1051-1063. doi: 10.1093/emboj/20.5.1051

Anisimova, M., and Gascuel, O. (2006). Approximate likelihood-ratio test for branches: a fast, accurate, and powerful alternative. Syst. Biol. 55, 539-552. doi: $10.1080 / 10635150600755453$

Batistič, O. (2012). Genomics and localization of the Arabidopsis DHHC-cysteinerich domain S-acyltransferase protein family. Plant Physiol. 160, 1597-1612. doi: 10.1104/pp.112.203968

Batistic, O., Kim, K. N., Kleist, T., Kudla, J., and Luan, S. (2011). "The CBL-CIPK network for decoding calcium signals in plants," in Coding and Decoding of Calcium Signals in Plants, ed S. Luan (New York, NY: Springer), 235-258. doi: 10.1007/978-3-642-20829-4_12

Batistič, O., and Kudla, J. (2009). Plant calcineurin B-like proteins and their interacting protein kinases. Biochim. Biophys. Acta 1793, 985-992. doi: 10.1016/j.bbamcr.2008.10.006

Batistic, O., Rehers, M., Akerman, A., Schlü, O. K., Steinhorst, L., Yalovsky, S., et al. (2012). S-acylation-dependent association of the calcium sensor CBL2 with the vacuolar membrane is essential for proper abscisic acid responses. Cell Res. 22, 1155-1168. doi: 10.1038/cr.2012.71

Batistic, O., Sorek, N., Schü, O. S., Yalovsky, S., and Kudla, J. (2008). Dual fatty acyl modification determines the localization and plasma membrane targeting of CBL/CIPK Ca ${ }^{2+}$ signaling complexes in Arabidopsis. Plant Cell 20, 1346-1362. doi: 10.1105/tpc.108.058123

Batistic, O., Waadt, R., Steinhorst, L., Held, K., and Kudla, J. (2010). CBL-mediated targeting of CIPKs facilitates the decoding of calcium signals emanating from distinct cellular stores. Plant J. 61, 211-222. doi: 10.1111/j.1365313X.2009.04045.x

Bhatnagar, R. S., and Gordon, J. I. (1997). Understanding covalent modifications of proteins by lipids: where cell biology and biophysics mingle. Trends Cell Biol. 7, 14-20. doi: 10.1016/S0962-8924(97)10044-7

Bijlmakers, M.-J., and Marsh, M. (2003). The on-iff story of protein palmitoylation. Trends Cell Biol. 13, 32-42. doi: 10.1016/S0962-8924(02)00008-9

Bowman, J. L., Floyd, S. K., and Sakakibara, K. (2007). Green genescomparative genomics of the green branch of life. Cell 129, 229-234. doi: 10.1016/j.cell.2007.04.004
Chang, S., Puryear, J., and Cairney, J. (1993). A simple and efficient method for isolating RNA from pine trees. Plant Mol. Biol. Rep. 11, 113-116. doi: 10.1007/BF02670468

Cheong, Y. H., Pandey, G. K., Grant, J. J., Batistic, O., Li, L., Kim, B., et al. (2007). Two calcineurin B-like calcium sensors, interacting with protein kinase CIPK23, regulate leaf transpiration and root potassium uptake in Arabidopsis. Plant J. 52, 223-239. doi: 10.1111/j.1365-313X.2007.03236.x

Clapham, D. E. (2007). Calcium signaling. Cell 131, 1047-1058. doi: 10.1016/j.cell.2007.11.028

Cui, L., Wall, P. K., Leebens-Mack, J. H., Lindsay, B. G., Soltis, D. E., Doyle, J. J., et al. (2006). Widespread genome duplications throughout the history of flowering plants. Genome Res. 16, 738-749. doi: 10.1101/gr.4825606

Cyert, M. S. (2001). Genetic analysis of calmodulin and its targets in Saccharomyces cerevisiae. Annu. Rev. Genet. 35, 647-672. doi: 10.1146/annurev.genet.35.102401.091302

D’Angelo, C., Weinl, S., Batistic, O., Pandey, G. K., Cheong, Y. H., Schüngel, S., et al. (2006). Alternative complex formation of the $\mathrm{Ca}^{2+}$-regulated protein kinase CIPK1 controls abscisic acid-dependent and independent stress responses in Arabidopsis. Plant J. 48, 857-872. doi: 10.1111/j.1365-313X.2006.02921.x

Dunphy, J. T., and Linder, M. E. (1998). Signalling functions of protein palmitoylation. Biochim. Biophys. Acta 1436, 245-261. doi: 10.1016/S0005-2760(98) 00130-1

Eisen, J. A., and Wu, M. (2002). Phylogenetic analysis and gene functional predictions: phylogenomics in action. Theor. Popul. Biol. 61, 481-487. doi: 10.1006/tpbi.2002.1594

Ellegren, H. (2004). Microsatellites: simple sequences with complex evolution. Nat. Rev. Genet. 5, 435-445. doi: 10.1038/nrg1348

Engelman, D. M. (2005). Membranes are more mosaic than fluid. Nature 438, 578-580. doi: 10.1038/nature04394

Evans, N. H., McAinsh, M. R., and Hetherington, A. M. (2001). Calcium oscillations in higher plants. Curr. Opin. Plant Biol. 4, 415-420. doi: 10.1016/S13695266(00)00194-1

Filichkin, S. A., Priest, H. D., Givan, S. A., Shen, R., Bryant, D. W., Fox, S. E., et al. (2010). Genome-wide mapping of alternative splicing in Arabidopsis thaliana. Genome Res. 20, 45-58. doi: 10.1101/gr.093302.109

Fogelson, A. L., and Zucker, R. S. (1985). Presynaptic calcium diffusion from various arrays of single channels. Implications for transmitter release and synaptic facilitation. Biophys. J. 48, 1003-1017. doi: 10.1016/S0006-3495(85)83863-7

Fraile-Escanciano, A., Kamisugi, Y., Cuming, A. C., Rodríscan-Navarro, A., and Benito, B. (2010). The SOS1 transporter of Physcomitrella patens mediates sodium efflux in planta. New Phytol. 188, 750-761. doi: 10.1111/j.14698137.2010.03405.x

Gilroy, S., Bethke, P. C., and Jones, R. L. (1993). Calcium homeostasis in plants. J. Cell Sci. 106, 453-461.

Graham, L. E. (1996). Green algae to land plants: an evolutionary transition. J. Plant Res. 109, 241-251. doi: 10.1007/BF02344471

Guindon, S., Delsuc, F., Dufayard, J. F., and Gascuel, O. (2009). "Estimating maximum likelihood phylogenies with PhyML," in Bioinformatics for DNA Sequence Analysis ed D. Posada (New York, NY: Springer), 113-137. doi: 10.1007/978-159745-251-9_6

Guo, Y., Halfter, U., Ishitani, M., and Zhu, J. K. (2001). Molecular characterization of functional domains in the protein kinase SOS2 that is required for plant salt tolerance. Plant Cell 13, 1383-1400. doi: 10.1105/tpc.13.6.1383

Hemsley, P. A., and Grierson, C. S. (2008). Multiple roles for protein palmitoylation in plants. Trends Plant Sci. 13, 295-302. doi: 10.1016/j.tplants.2008.04.006

Hirotsune, S., Yoshida, N., Chen, A., Garrett, L., Sugiyama, F., Takahashi, S., et al. (2003). An expressed pseudogene regulates the messenger-RNA stability of its homologous coding gene. Nature 423, 91-96. doi: 10.1038/nature01535

Ho, C. H., Lin, S. H., Hu, H. C., and Tsay, Y. F. (2009). CHL1 functions as a nitrate sensor in plants. Cell 138, 1184-1194. doi: 10.1016/j.cell.2009.07.004

Hrabak, E. M., Chan, C. W. M., Gribskov, M., Harper, J. F., Choi, J. H., Halford, N., et al. (2003). The Arabidopsis CDPK-SnRK superfamily of protein kinases. Plant Physiol. 132, 666-680. doi: 10.1104/pp.102.011999

Hu, H., Wang, Y., and Tsay, Y. (2009). AtCIPK8, a CBL-interacting protein kinase, regulates the low-affinity phase of the primary nitrate response. Plant J. 57, 264-278. doi: 10.1111/j.1365-313X.2008.03685.x

Jain, E., Bairoch, A., Duvaud, S., Phan, I., Redaschi, N., Suzek, B. E., et al. (2009). Infrastructure for the life sciences: design and implementation of the UniProt website. BMC Bioinformatics 10:136. doi: 10.1186/1471-2105-10-136 
Jeffares, D. C., Mourier, T., and Penny, D. (2006). The biology of intron gain and loss. Trends Genet. 22, 16-22. doi: 10.1016/j.tig.2005.10.006

Jiao, Y., Wickett, N. J., Ayyampalayam, S., Chanderbali, A. S., Landherr, L., Ralph, P. E., et al. (2011). Ancestral polyploidy in seed plants and angiosperms. Nature 473, 97-100. doi: 10.1038/nature09916

Katoh, K., Kuma, K., and Miyata, T. (2002). MAFFT: a novel method for rapid multiple sequence alignment based on fast Fourier transform. Nucleic Acids Res. 30, 2059-3066. doi: 10.1093/nar/gkf436

Kenrick, P., and Crane, P. R. (1997). The origin and early evolution of plants on land. Nature 389, 33-39. doi: 10.1038/37918

Kim, B., Waadt, R., Cheong, Y. H., Pandey, G. K., Dominguez-Solis, J. R., Schüolis, S., et al. (2007). The calcium sensor CBL10 mediates salt tolerance by regulating ion homeostasis in Arabidopsis. Plant J. 52, 473-484. doi: 10.1111/j.1365-313X.2007.03249.x

Kolukisaoglu, Ü., Weinl, S., Blazevic, D., Batistic, O., and Kudla, J. (2004). Calcium sensors and their interacting protein kinases: genomics of the Arabidopsis and rice CBL-CIPK signaling networks. Plant Physiol. 134, 43-58. doi: 10.1104/pp.103.033068

Korneev, S. A., Park, J. H., and O'Shea, M. (1999). Neuronal expression of neural nitric oxide synthase (nNOS) protein is suppressed by an antisense RNA transcribed from an NOS pseudogene. J. Neurosci. 19, 7711-7720.

Kudla, J., Xu, Q., Harter, K., Gruissem, W., and Luan, S. (1999). Genes for calcineurin B-like proteins in Arabidopsis are differentially regulated by stress signals. Proc. Natl. Acad. Sci. U.S.A. 96, 4718-4723. doi: 10.1073/pnas.96.8.4718

Levental, I., Grzybek, M., and Simons, K. (2010). Greasing their way: lipid modifications determine protein association with membrane rafts. Biochemistry 49, 6305-6316. doi: 10.1021/bi100882y

Lewis, L. A., and McCourt, R. M. (2004). Green algae and the origin of land plants. Am. J. Bot. 91, 1535-1556. doi: 10.3732/ajb.91.10.1535

Li, C., Pan, W., Braunewell, K. H., and Ames, J. B. (2011). Structural analysis of $\mathrm{Mg}^{2+}$ and $\mathrm{Ca}^{2+}$ binding, myristoylation, and dimerization of the neuronal calcium sensor and visinin-like protein 1 (VILIP-1). J. Biol. Chem. 286, 6354-6366. doi: 10.1074/jbc.M110.173724

Li, L., Kim, B. G., Cheong, Y. H., Pandey, G. K., and Luan, S. (2006). A Ca ${ }^{2+}$ signaling pathway regulates a $\mathrm{K}^{+}$channel for low-K response in Arabidopsis. Proc. Natl. Acad. Sci. U.S.A. 103, 12625-12630. doi: 10.1073/pnas.0605129103

Liu, J., Ishitani, M., Halfter, U., Kim, C.-S., and Zhu, J.-K. (2000). The Arabidopsis thaliana SOS2 gene encodes a protein kinase that is required for salt tolerance. Proc. Natl. Acad. Sci. U.S.A. 97, 3730-3734. doi: 10.1073/pnas.97.7.3730

Liu, J., and Zhu, J. K. (1998). A calcium sensor homolog required for plant salt tolerance. Science 280, 1943-1945. doi: 10.1126/science.280.5371.1943

Luan, S. (2009). The CBLsor homolog requireant calcium signaling. Trends Plant Sci. 14, 37-42. doi: 10.1016/j.tplants.2008.10.005

Luan, S., Kudla, J., Rodriguez-Concepcion, M., Yalovsky, S., and Gruissem, W. (2002). Calmodulins and calcineurin B-like proteins: calcium sensors for specific signal response coupling in plants. Plant Cell 14(suppl. 1), S389-S400. doi: 10.1105/tpc.001115

Martínez-Atienza, J., Jiang, X., Garciadeblas, B., Mendoza, I., Zhu, J. K., Pardo, J. M. et al. (2007). Conservation of the salt overly sensitive pathway in rice. Plant Physiol. 143, 1001-1012. doi: 10.1104/pp.106.092635

McCormack, E., and Braam, J. (2003). Calmodulins and related potential calcium sensors of Arabidopsis. New Phytol. 159, 585-598. doi: 10.1046/j.14698137.2003.00845.x

McCormack, E., Tsai, Y. C., and Braam, J. (2005). Handling calcium signaling: Arabidopsis CaMs and CMLs. Trends Plant Sci. 10, 383-389. doi: 10.1016/j.tplants.2005.07.001

Mumby, S. M. (1997). Reversible palmitoylation of signaling proteins. Curr. Opin. Cell Biol. 9, 148-154. doi: 10.1016/S0955-0674(97)80056-7

Nagae, M., Nozawa, A., Koizumi, N., Sano, H., Hashimoto, H., Sato, M., et al. (2003). The crystal structure of the novel calcium-binding protein AtCBL2 from Arabidopsis thaliana. J. Biol. Chem. 278, 42240-42246. doi: 10.1074/jbc.M303630200

Pandey, G. K., Cheong, Y. H., Kim, B. G., Grant, J. J., Li, L., and Luan, S. (2007). CIPK9: a calcium sensor-interacting protein kinase required for lowpotassium tolerance in Arabidopsis. Cell Res. 17, 411-421. doi: 10.1038/cr. 2007.39

Pandey, G. K., Grant, J. J., Cheong, Y. H., Kim, B. G., Li le, G., and Luan, S. (2008). Calcineurin-B-like protein CBL9 interacts with target kinase CIPK3 in the regulation of ABA response in seed germination. Mol. Plant 1, 238-248. doi: $10.1093 / \mathrm{mp} / \mathrm{ssn} 003$

Pittermann, J. (2010). The evolution of water transport in plants: an integrated approach. Geobiology 8, 112-139. doi: 10.1111/j.1472-4669.2010.00232.x

Quan, R., Lin, H., Mendoza, I., Zhang, Y., Cao, W., Yang, Y., et al. (2007). SCABP8/CBL10, a putative calcium sensor, interacts with the protein kinase SOS2 to protect Arabidopsis shoots from salt stress. Plant Cell 19, 1415-1431. doi: 10.1105/tpc.106.042291

Quintero, F. J., Martinez-Atienza, J., Villalta, I., Jiang, X., Kim, W. Y., Ali, Z., et al. (2011). Activation of the plasma membrane $\mathrm{Na} / \mathrm{H}$ antiporter Salt-Overly-Sensitive 1 (SOS1) by phosphorylation of an auto-inhibitory C-terminal domain. Proc. Natl. Acad. Sci. U.S.A. 108, 2611-2616. doi: 10.1073/pnas.1018921108

Ren, X., Qi, G., Feng, H., Zhao, S., Zhao, S., Wang, Y., et al. (2013). Calcineurin Blike protein CBL10 directly interacts with AKT1 and modulates $\mathrm{K}+$ homeostasis in Arabidopsis. Plant J. 74, 258-266. doi: 10.1111/tpj.12123

Rensing, S. A., Beike, A. K., and Lang, D. (2013). "Evolutionary importance of generative polyploidy for genome evolution of haploid-dominant land plants," in Plant Genome Diversity, Vol. 2, eds I. J. Leitch, J. Greilhuber, J. Dolezel and J. Wendel (New York, NY: Springer), 295-305. doi: 10.1007/978-3-7091-11604_18

Rensing, S. A., Ick, J., Fawcett, J. A., Lang, D., Zimmer, A., Van de Peer, Y., et al. (2007). An ancient genome duplication contributed to the abundance of metabolic genes in the moss Physcomitrella patens. BMC Evol. Biol. 7:130. doi: 10.1186/1471-2148-7-130

Rensing, S. A., Lang, D., Zimmer, A. D., Terry, A., Salamov, A., Shapiro, H., et al. (2008). The Physcomitrella genome reveals evolutionary insights into the conquest of land by plants. Science 319, 64-69. doi: 10.1126/science.1150646

Roberts, W. M. (1994). Localization of calcium signals by a mobile calcium buffer in frog saccular hair cells. J. Neurosci. 14, 3246-3262.

Shi, H., Ishitani, M., Kim, C., and Zhu, J.-K. (2000). The Arabidopsis thaliana salt tolerance gene SOS1 encodes a putative $\mathrm{Na}^{+} / \mathrm{H}^{+}$antiporter. Proc. Natl. Acad. Sci. U.S.A. 97, 6896-6901. doi: 10.1073/pnas.120170197

Shi, J., Kim, K. N., Ritz, O., Albrecht, V., Gupta, R., Harter, K., et al. (1999). Novel protein kinases associated with calcineurin B-like calcium sensors in Arabidopsis. Plant Cell 11, 2393-2405. doi: 10.1105/tpc.11.12.2393

Sjölander, K. (2004). Phylogenomic inference of protein molecular function: advances and challenges. Bioinformatics 20, 170-179. doi: 10.1093/bioinformatics/bth021

Stanke, M., Steinkamp, R., Waack, S., and Morgenstern, B. (2004). AUGUSTUS: a web server for gene finding in eukaryotes. Nucleic Acids Res. 32, W309-W312. doi: 10.1093/nar/gkh379

Tam, O. H., Aravin, A. A., Stein, P., Girard, A., Murchison, E. P., Cheloufi, S., et al. (2008). Pseudogene-derived small interfering RNAs regulate gene expression in mouse oocytes. Nature 453, 534-538. doi: 10.1038/nature06904

Tang, R. J., Liu, H., Bao, Y., Lv, Q. D., Yang, L., and Zhang, H. X. (2010). The woody plant poplar has a functionally conserved salt overly sensitive pathway in response to salinity stress. Plant Mol. Biol. 74, 367-380. doi: 10.1007/s11103010-9680-x

Tang, R. J., Liu, H., Yang, Y., Yang, L., Gao, X. S., Garcia, V. J., et al. (2012). Tonoplast calcium sensors CBL2 and CBL3 control plant growth and ion homeostasis through regulating V-ATPase activity in Arabidopsis. Cell Res. 22, 1650-1665. doi: $10.1038 / \mathrm{cr} .2012 .161$

Tang, R. J., Yang, Y., Yang, L., Liu, H., Wang, C. T., Meng-Meng, Y., et al. (2013). Poplar calcineurin B-like proteins PtCBL10A and PtCBL10B regulate shoot salt tolerance through interaction with PtSOS2 in the vacuolar membrane. Plant Cell Environ. 37, 573-588. doi: 10.1111/pce.12178

Timme, R., and Delwiche, C. (2010). Uncovering the evolutionary origin of plant molecular processes: comparison of Coleochaete (Coleochaetales) and Spirogyra (Zygnematales) transcriptomes. BMC Plant Biol. 10:96. doi: 10.1186/14712229-10-96

Timme, R. E., Bachvaroff, T. R., and Delwiche, C. F. (2012). Broad phylogenomic sampling and the sister lineage of land plants. PLOS ONE 7:e29696. doi: 10.1371/journal.pone.0029696

Verret, F., Wheeler, G., Taylor, A. R., Farnham, G., and Brownlee, C. (2010). Calcium channels in photosynthetic eukaryotes: implications for evolution of calcium-based signalling. New Phytol. 187, 23-43. doi: 10.1111/j.14698137.2010.03271.x 
Weinl, S., and Kudla, J. (2009). The CBL-CIPK $\mathrm{Ca}^{2+}$-decoding network: function and perspectives. New Phytol. 184, 517-528. doi: 10.1111/j.14698137.2009.02938.x

Wheeler, G. L., and Brownlee, C. (2008). $\mathrm{Ca}^{2+}$ signalling in plants and green algaeand perspectives. Trends Plant Sci. 13, 506-514. doi: 10.1016/j.tplants.2008.06.004

Witman, G. B. (1993). Chlamydomonas phototaxis. Trends Cell biol. 3, 403-408. doi: 10.1016/0962-8924(93)90091-E

Xu, J., Li, H. D., Chen, L. Q., Wang, Y., Liu, L. L., He, L., et al. (2006). A protein kinase, interacting with two calcineurin B-like proteins, regulates $\mathrm{K}^{+}$transporter AKT1 in Arabidopsis. Cell 125, 1347-1360. doi: 10.1016/j.cell.2006.06.011

Zimmer, A. D., Lang, D., Buchta, K., Rombauts, S., Nishiyama, T., Hasebe, M., et al. (2013). Reannotation and extended community resources for the genome of the non-seed plant Physcomitrella patens provide insights into the evolution of plant gene structures and functions. BMC Genomics 14:498. doi: 10.1186/1471-216414-498
Conflict of Interest Statement: The authors declare that the research was conducted in the absence of any commercial or financial relationships that could be construed as a potential conflict of interest.

Received: 31 January 2014; accepted: 21 April 2014; published online: 14 May 2014. Citation: Kleist TJ, Spencley AL and Luan S (2014) Comparative phylogenomics of the CBL-CIPK calcium-decoding network in the moss Physcomitrella, Arabidopsis, and other green lineages. Front. Plant Sci. 5:187. doi: 10.3389/fpls.2014.00187

This article was submitted to Plant Genetics and Genomics, a section of the journal Frontiers in Plant Science.

Copyright (C) 2014 Kleist, Spencley and Luan. This is an open-access article distributed under the terms of the Creative Commons Attribution License (CC BY). The use, distribution or reproduction in other forums is permitted, provided the original author(s) or licensor are credited and that the original publication in this journal is cited, in accordance with accepted academic practice. No use, distribution or reproduction is permitted which does not comply with these terms. 